\title{
Invariants of pseudogroup actions: Homological methods and Finiteness theorem
}

\author{
Boris Kruglikov and Valentin Lychagin \\ Mat-Stat. Dept., University of Troms $\varnothing$, Norway
}

September 26, 2018

\begin{abstract}
We study the equivalence problem of submanifolds with respect to a transitive pseudogroup action. The corresponding differential invariants are determined via formal theory and lead to the notions of $l$-variants and $l$-covariants, even in the case of non-integrable pseudogroup. Their calculation is based on the cohomological machinery: We introduce a complex for covariants, define their cohomology and prove the finiteness theorem. This implies the well-known Lie-Tresse theorem about differential invariants. We also generalize this theorem to the case of pseudogroup action on differential equations. ${ }^{1}$
\end{abstract}

\section{Introduction}

Transformation groups were introduced by S. Lie [Li1] in his study of invariants of differential equations. More formal and general notion of pseudogroup was developed by E. Cartan C1. These infinite groups of Lie and Cartan (see also $[\mathrm{H}, \operatorname{Tr} 1, \mathrm{~V}]$ ) play nowadays a central role in geometry and analysis.

A pseudogroup $G \subset \operatorname{Diff}_{\text {loc }}(M)$ acting on a manifold $M$ consists of a collection of local diffeomorphisms $\varphi$, each bearing own domain of definition $\operatorname{dom}(\varphi)$ and range $\operatorname{im}(\varphi)$, that satisfies the following properties:

1. $\operatorname{id}_{M} \in G$ and $\operatorname{dom}\left(\operatorname{id}_{M}\right)=\operatorname{im}\left(\operatorname{id}_{M}\right)=M$,

2. If $\varphi, \psi \in G$, then $\varphi \circ \psi \in G$ whenever $\operatorname{dom}(\varphi) \subset \operatorname{im}(\psi)$,

3. If $\varphi \in G$, then $\varphi^{-1} \in G$ and $\operatorname{dom}\left(\varphi^{-1}\right)=\operatorname{im}(\varphi)$,

4. $\varphi \in G$ iff for every open subset $U \in \operatorname{dom}(\varphi)$ the restriction $\left.\varphi\right|_{U} \in G$,

\footnotetext{
${ }^{1}$ MSC numbers: 53A55, 58H10; 35A30, 58A20. Keywords: pseudogroup, differential invariants, Tresse derivative, equivalence, Lie equation, Spencer cohomology.
} 
5. The pseudogroup is of order $l$ if this is the minimal number such that $\varphi \in G$ whenever for each point $a \in \operatorname{dom}(\varphi)$ the $l$-jet is admissible: $[\varphi]_{a}^{l} \in G^{l}$.

The latter property means that a pseudogroup is defined by differential equations of maximal order $l$ and will be explained below. It uses the infinitesimal language. In fact, for most infinite pseudogroups the only comprehensive way to introduce the notion of continuity is via the prolongation technique.

A transformation $\varphi \in G$ defines a map (l-th prolongation) of the space of jets of codimension $r$ submanifolds $\varphi_{(l)}: J_{r}^{l}(M) \rightarrow J_{r}^{l}(M)$, which obeys the following property:

$$
\left(\varphi \circ \psi^{-1}\right)_{(l)}=\varphi_{(l)} \circ \psi_{(l)}^{-1} .
$$

This property is fundamental and being coupled with the formal theory of differential equations leads to a deep understanding of pseudogroups, cf. [E, Lib, SS.

In this paper we develop a more general notion of infinitesimal pseudogroup. First of all, we don't require a pseudogroup to be integrable, and so consider an $l$-pseudogroup as a transformation group in finite jets. Even such finite order pseudogroups are important in producing invariants for differential equations and curvatures for geometric structures.

Next, we consider pseudogroups acting on differential equations and this relates the theory with the original approach of S. Lie, which is now called the theory of symmetries and is extensively used in explicit integration of differential equation. Using this technique the known invariant differential operators, connections etc can be obtained.

Finally we do no require that pseudogroups act effectively. In our approach the stationary sub-pseudogroups appear naturally. This is convenient for applications, since we can consider then general representations.

Formal integrability criterion for infinitesimal pseudogroups is based on the well-developed algebraic machinery, described in the paper. The passage from formal integrability to the local one is not automatic and is generically wrong. However the former implies the latter in the following cases:

- Finite type pseudogroups (the symbol $\mathfrak{g}^{k} \equiv 0$ for big $k$ ). This condition implies that the integrated pseudogroup is finite-dimensional (Lie group).

- Analytic pseudogroups. It is a consequence of Cartan-Kähler theorem, which holds for general differential equations [M] KLV].

- Elliptic pseudogroups of analytic type, see $[\underline{\mathrm{S}}] \mathbf{M}]$.

- Transitive flat pseudogroups, see [BM] $\mathrm{P}$.

Only in special cases the global integrability (or equivalence) problem can be handled, see [S] GS2, Ta.

Like the classical invariant theory, the theory of invariants of pseudogroup actions exists. For Lie pseudogroups this is the well-known differential invariants theory. Generally the number of these differential invariants is infinite (the opposite case is of much importance, was discussed in our preceding paper 
KL2 and will be reviewed and continued here). But the whole algebra can be finitely generated (under certain regularity assumptions). This can be done via Lie approach with a finite number of invariant differentiations generating all invariants from a finite number of them (Li3, Li4])) or with Tresse method of differentiating some invariants by the others ([Tr1]).

In this paper we address the question of calculation of the differential invariants and estimation of their number. We develop the theory of pseudogroups actions on submanifolds, which are subject to certain differential relations. In other words, we consider the action of a pseudogroup on a system of differential equations, which we naturally identify with a submanifold in the space of jets.

On the level of finite jets we are lead to what we call $l$-covariants. Their calculus is governed by certain cohomology theory similar to the formal Spencer cohomology. We exploit this in relation to the problem of equivalence of submanifolds under the action.

Our approach gives the finiteness theorem for the cohomology of covariants and this in turn implies Lie ([Li2, Li5]) and Tresse ([Tr1]) theorems (proved by Kumpera Kum, see also modifications in $\mathrm{Ov}, \mathrm{Ol}, \mathrm{MMR}$ ) and their generalization for the action of pseudogroups on differential equations (note that in Kumpera's approach the pseudogroup is integrable and he considers the integrated sheaf of vector fields, while we start with general pseudogroup and calculate precisely how many invariants we need on each jet level).

Lie pseudogroups consist of pseudo-automorphisms of geometric structures. We provide a series of calculations for them. An important class form the symmetries of differential equations, realized as transformations preserving the Cartan distribution on the equation. They are very useful for integration of the given equation.

\section{Pseudogroups and equivalence}

In this section we discuss the general introduction of pseudogroups, developing the ideas of [GS2, Kur, [S, Ta]. This approach was proposed in [KL2].

\subsection{Formal pseudogroups}

Let $M$ be a smooth manifold and $J_{r}^{l}(M)$ be the corresponding jet space. Its points $a_{l}$ are the $l$-jets $[N]_{a}^{l}$ of submanifolds $N \subset M$ of codimension $r$ at $a \in N$.

Denote the natural projections by $\rho_{i, j}: J_{r}^{i}(M) \rightarrow J_{r}^{j}(M), i \geq j$. It $j \geq 1$ the fibers bear a canonical affine structure $([\mathrm{KLV}, \mathrm{Ly}])$, associated with the vector structure, described below. It is sufficient to specify it for $\mathfrak{F}\left(a_{l-1}\right)=\rho_{l, l-1}^{-1}\left(a_{l-1}\right)$.

Denote $\mathfrak{t}_{a}=T_{a} N=[N]_{a}^{1}$ and $\mathfrak{v}_{a}=T_{a} M / T_{a} N$. Let $a_{l} \in J_{r}^{l}(M), a_{l-1}=$ $\rho_{l, l-1}\left(a_{l}\right)$. Then $T_{a_{l}} \mathfrak{F}\left(a_{l-1}\right) \simeq S^{l} \mathfrak{t}_{a}^{*} \otimes \mathfrak{v}_{a}$ and we get the exact sequence:

$$
0 \rightarrow S^{l} \mathfrak{t}_{a}^{*} \otimes \mathfrak{v}_{a} \rightarrow T_{a_{l}} J_{r}^{l}(M) \stackrel{\left(\rho_{l, l-1}\right)_{*}}{\longrightarrow} T_{a_{l-1}} J_{r}^{l-1}(M) \rightarrow 0 .
$$

For a vector bundle $\rho: E \rightarrow B$ of rank $r$, the corresponding space of jets of sections $J^{l} \rho$ is an open subset in $J_{r}^{l}(E)$. In particular, we realize the jet space 
for maps $J^{l}(N, M)$. Denote by $D^{l}(M) \subset J^{l}(M, M)$ the open dense subset, consisting of the $l$-jets of local diffeomorphisms. Being equipped with the partially defined composition operation, it is an example of finite order pseudogroup.

To define this notion in general, recall some basic facts from the geometric theory of differential equations, see $\mathrm{KLV}, \mathrm{Ly}, \mathrm{Gu}$ and Appendix $\mathrm{A}$ for details. The prolongation of differential equation $\mathcal{E} \subset J_{r}^{l}(M)$ is defined as

$$
\begin{aligned}
& \mathcal{E}^{(1)}=\left\{a_{l+1}=[N]_{a}^{l+1} \mid \text { for } N \subset M \text { if codimension } r\right. \text { the jet-extension } \\
& \left.\qquad j_{l}(N) \subset J_{r}^{l}(M) \text { is tangent to } \mathcal{E} \text { at } a_{l}\right\} \subset J_{r}^{l+1}(M) .
\end{aligned}
$$

This can be equivalently written as $\mathcal{E}^{(1)}=\left\{a_{l+1} \mid L\left(a_{l+1}\right) \subset T_{a_{l}} \mathcal{E}\right\}$, where for $a_{l+1}=[N]_{a}^{l+1}$ we set: $L\left(a_{l+1}\right)=T_{a_{l}} j_{l}(N), a_{l}=\rho_{l+1, l}\left(a_{l+1}\right)$.

The other prolongations are defined inductively: $\mathcal{E}^{(i)}=\left(\mathcal{E}^{(i-1)}\right)^{(1)}$.

Definition 1. An l-pseudogroup is a collection of (non-empty) subbundles $G^{j} \subset$ $D^{j}(M), 0<j \leq l$, such that the following properties are satisfied:

1. If $\varphi_{j}, \psi_{j} \in G^{j}$, then $\varphi_{j} \circ \psi_{j}^{-1} \in G^{j}$ whenever defined,

2. The map $\rho_{j, j-1}: G^{j} \rightarrow G^{j-1}$ is a bundle for every $j \leq l$.

As usual in the differential equations theory we assume $G^{0}=D^{0}(M)=M \times M$, which is equivalent to transitivity of the pseudogroup action.

An l-pseudogroup is called l-integrable if $G^{j} \subset\left(G^{j-1}\right)^{(1)}$ for all $0<j \leq l$.

Note that assumption 1 implies that $\operatorname{id}_{M}^{j} \in G^{j}$ and $\varphi_{j} \in G^{j} \Rightarrow \varphi_{j}^{-1} \in G^{j}$.

Pseudogroups $G=\left\{G^{j}\right\}_{j=1}^{l}$ defined by this approach can be studied for integrability by the standard prolongation-projection method, see GS1, GS2, Kur, KLV, S, Ta and Appendix \$

Denote $G_{a, b}^{j}=\left\{\varphi_{j} \in G^{j} \mid \varphi_{0}(a)=b\right\}, G_{a}^{j}=G_{a, a}^{j}$ - the subgroup of $G^{j}$ and $\mathfrak{G}_{a}^{j}=\operatorname{Ker}\left[\rho_{j, j-1}: G_{a}^{j} \rightarrow G_{a}^{j-1}\right]$ - its (normal) subgroup, which is abelian for $j>1$ and for $j=1: \mathfrak{G}_{a}^{1}=G_{a}^{1} \subset \operatorname{Gl}\left(T_{a} M\right)$.

Definition 2. Let $\varphi_{j} \in G^{j}$ be a point and $\rho_{j, 0}\left(\varphi_{j}\right)=(a, b) \in M \times M$. The symbol of the pseudogroup $G$ is given by:

$$
\mathfrak{g}^{j}\left(\varphi_{j}\right)=\operatorname{Ker}\left[\left(\rho_{j, j-1}\right)_{*}: T_{\varphi_{j}} G^{j} \rightarrow T_{\varphi_{j-1}} G^{j-1}\right] .
$$

It can be viewed as a subspace $\mathfrak{g}^{j}\left(\varphi_{j}\right) \subset S^{j}\left(T_{a}^{*} M\right) \otimes T_{b} M \stackrel{1 \otimes \varphi_{1}^{-1}}{\simeq} S^{j}\left(T_{a}^{*} M\right) \otimes T_{a} M$, and in the last form is identified with the Lie algebra $\mathfrak{g}_{a}^{j}$ of the Lie group $\mathfrak{G}_{a}^{j}$.

An $l$-pseudogroup $G$ is called formally integrable if it is $l$-integrable, for every $j>l$ the prolongation $G^{j}=\left(G^{l}\right)^{(j-l)}$ exists, are $j$-pseudogroups and the projections $\rho_{j, j-1}: G^{j} \rightarrow G^{j-1}$ are vector bundles.

Similar to the differential equations theory ( $\mathrm{Go}, \mathrm{Gu}, \underline{\mathrm{S}})$ ), a criterion of formal integrability can be formulated in terms of the Spencer $\delta$-complex:

$$
0 \rightarrow \mathfrak{g}_{a}^{l} \stackrel{\delta}{\rightarrow} \mathfrak{g}_{a}^{l-1} \otimes T_{a}^{*} M \stackrel{\delta}{\rightarrow} \ldots \stackrel{\delta}{\rightarrow} \mathfrak{g}_{a}^{l-j} \otimes \Lambda^{j} T_{a}^{*} M \stackrel{\delta}{\rightarrow} \ldots
$$


Its bi-graded cohomology groups are denoted by $H^{l-j, j}(G)$ or $H^{l-j, j}(\mathfrak{g})$. Poincaré $\delta$-lemma states that $\operatorname{dim} H^{*, *}(G)<\infty$, so that all cohomology groups $H^{i, j}(\mathfrak{g})$ eventually vanish. But some groups are non-zero.

The obstructions to formal integrability of the $l$-pseudogroup $G$, considered as a differential equation, are some elements $W_{j}(G) \in H^{j-1,2}(G)$, called Weyl tensors (or curvatures), defined via the jet-spaces geometry. We do not need their precise form here and so refer for the definition to $\mathrm{Ly}$.

Theorem 1. Let $G$ be an l-pseudogroup. Suppose the symbols $\mathfrak{g}^{j}$ over $G^{l}$ form a vector bundle and all the Weyl tensors $W_{j}$ vanish identically for all $j \geq l$. Then the pseudogroup is formally integrable.

Proof. The hypotheses imply integrability of $G$ as a differential equation, see $\left[\mathrm{Ly}\right.$. We need to check that the obtained system $\left\{G^{j}\right\}_{j=0}^{\infty}$ is a pseudogroup, i.e. to check all the requirements of definition 1.

Let $G^{j+1}=\left(G^{j}\right)^{(1)}$. Obviously, the unit is in $G^{j+1}$. Let $\varphi_{j+1} \in G_{a, b}^{j+1}$, $\psi_{j+1} \in G_{b, c}^{j+1}$ and $\chi_{j+1}=\psi_{j+1} \circ \varphi_{j+1}$. We need to show that $\chi_{j+1} \in G^{j+1}$. This is equivalent to $L\left(\chi_{j+1}\right) \subset T_{\chi_{j}} G^{j}$.

To prove the inclusion consider the multiplication operator $m_{j}: G^{j} \times G^{j} \rightarrow$ $G^{j}$. It has the differential:

$$
T_{\psi_{j}} G^{j} \oplus T_{\varphi_{j}} G^{j} \stackrel{d m_{j}}{\longrightarrow} T_{\chi_{j}} G^{j} .
$$

The two summands on the left contain the subspaces $L\left(\psi_{j+1}\right)$ and $L\left(\varphi_{j+1}\right)$ respectively. But

$$
L\left(\psi_{j+1}\right) \oplus L\left(\varphi_{j+1}\right) \stackrel{d m_{j}}{\longrightarrow} L\left(\psi_{j+1} \varphi_{j+1}\right)
$$

for any $\varphi_{j+1}, \psi_{j+1} \in D^{j+1}(M)$ such that the composition is defined. Moreover the multiplication operator with one fixed argument is invertible. So the above arguments yield that $\varphi_{j+1} \in G^{j+1}$ implies $\varphi_{j+1}^{-1} \in G^{j+1}$. The claim follows.

An $l$-pseudogroup $G$ is called $q$-acyclic if $H^{i, j}(G)=0$ for $i \geq l, 0 \leq j \leq$ $q$. An $\infty$-acyclic pseudogroup is called involutive. For such pseudogroups $G$ investigation of formal integrability involves only one obstruction $W_{l}(G)$.

If a pseudogroup $G$ is formally integrable we obtain its infinite prolongation

$$
G^{\infty}=\lim _{\text {proj }}\left(G^{l}, \rho_{l, l-1}\right),
$$

which is called infinitesimal or formal pseudogroup. If there is local integrability (smooth or analytic), as described in the introduction, we refer to the pseudogroup as to integrable.

Lie pseudogroups are characterized by the property that they can be restored from the corresponding equation on jets of vector fields, precisely in the way the Lie groups and algebras are related, see [KS]. The corresponding Lie equations for such pseudogroups are always linear. 
Example 1. The group of complex fractional-linear transformations of $S^{2}=$ $\mathbb{C} P^{1}$ (or real transformations of $S^{1}=\mathbb{R} P^{1}$ ) is an integrable pseudogroup of finite type and order 3 . In fact its Lie algebra is represented as the algebra of quadratic-polynomial vector fields on the line: $\mathfrak{g}=\left\{\xi=\left(c_{0}+c_{1} z+c_{2} z^{2}\right) \partial_{z}\right\}$.

Example 2. Consider the pseudogroup of local plane transformations

$$
(q, p) \mapsto\left(F(q), p / F^{\prime}(q)\right) .
$$

This is a Lie pseudogroup of infinite type and order 1 . Indeed, it consists of transformations from $T^{*} \mathbb{R}^{1} \simeq \mathbb{R}^{2}(q, p)$ preserving the Liouville form $p d q$. The generating field has the form $\xi=f(q) \partial_{q}-f^{\prime}(q) p \partial_{p}$. If we vary $F(q)$ in a finitedimensional subgroup of $\operatorname{Diff}\left(\mathbb{R}^{1}\right)$, the pseudogroup becomes of finite type.

Example 3. Let $\mathcal{E}$ be a geometric structure (Gu, Ly $)$ and $G$ be its Lie pseudogroup of the jets-automorphisms. If the structure $\mathcal{E}$ is integrable (flat), the pseudogroup is integrable as well. It can be of finite or infinite type depending on the geometric structure $(\underline{\mathrm{Ko}})$. It has the same order as the structure $\mathcal{E}$. When the geometric structure is non-integrable, the order of the pseudogroup $G$ can increase and it can readily be non-integrable (formally or locally).

One of the most important Lie pseudogroups consist of Lie transformations on the jet-space $M=J^{k} \pi$ of some bundle $\pi: E_{\pi} \rightarrow B$ ([KLV]). It has order 1 and infinite type. We will discuss this example in detail in 4 and Appendix B

\subsection{Pseudogroup action}

A pseudogroup $G$ is represented by the action on local submanifolds $N \subset M$ of codimension $r$. A formal pseudogroup acts on the space $J_{r}^{l}(M)$.

The equivalence problem is to realize when a submanifold $N_{1} \subset M$ can be transformed to a submanifold $N_{2} \subset M$ by a map $\varphi \in G$. For formal pseudogroups we consider the infinitesimal problem for $l$-jets and $l$-pseudogroups:

Definition 3. We say that l-jets of two submanifolds $N_{1}$ and $N_{2}$ at the points $a, b \in M$ are $G$-equivalent if $\varphi_{l}\left[N_{1}\right]_{a}^{l}=\left[N_{2}\right]_{b}^{l}$ for some $\varphi_{l} \in G_{a, b}^{l}$.

For transitive pseudogroups the equivalence problem reduces to the case $a=$ $b$. We assume this and begin subsequently equalizing the jets of submanifolds.

The pseudogroup $D^{l}(M)$ and hence $G^{l}$ act on the space $J_{r}^{l}(M)$ by the formula $\varphi_{(l)}:[N]_{a}^{l} \mapsto[\varphi(N)]_{\varphi(a)}^{l}$. These actions obey the relation: $\rho_{l, s} \circ \varphi_{(l)}=$ $\varphi_{(s)} \circ \rho_{l, s}$.

Consequently, the group $\mathfrak{G}_{a}^{l}$ acts on $\mathfrak{F}\left(a_{l-1}\right)$. For $l=1$ this action is generated by the linear collineations in the Grassmannians. The action is affine for $l>1$ :

$$
f \mapsto \lambda(\theta)+f, \quad f \in T_{a_{l}} \mathfrak{F}\left(a_{l-1}\right) .
$$

Here $\lambda$ is the induced linear representation of the Lie algebra $\mathfrak{g}_{a}^{l}$, which is naturally the restriction-factorization map:

$$
\lambda: S^{l} T_{a}^{*} M \otimes T_{a} M \rightarrow T_{a_{k}} \mathfrak{F}\left(a_{l-1}\right), \quad \theta \mapsto \bar{\theta} \in S^{l} \mathfrak{t}_{a}^{*} \otimes \mathfrak{v}_{a} .
$$


Thus the stabilizer of an element $a_{l} \in \mathfrak{F}\left(a_{l-1}\right)$ equals $\mathfrak{H}_{a}^{l}=\mathfrak{G}_{a}^{l} \cap \mathrm{St}_{a}^{l}$ in the case of the Lie group, or

$$
\mathfrak{h}_{a}^{l}=\mathfrak{g}_{a}^{l} \cap \mathrm{St}_{a}^{l}
$$

for the Lie algebra, where

$$
\mathrm{St}_{a}^{l}=\left(\operatorname{Ann} \mathfrak{t}_{a}\right) \circ_{\mathrm{sym}} S^{l-1} T_{a}^{*} M \otimes T_{a} M+S^{l} T_{a}^{*} M \otimes \mathfrak{t}_{a} .
$$

In particular, since the symbol of $D^{l}(M)$ acts transitively, we get:

$$
S^{l} T_{a}^{*} M \otimes T_{a} M / \mathrm{St}_{a}^{l} \simeq S^{l} \mathfrak{t}_{a}^{*} \otimes \mathfrak{v}_{a} .
$$

Remark 1. The group $\mathfrak{G}_{a}^{l}$ for $l>1$ is abelian, which reflect the affine property of the action, and so we can work only with Lie algebras. In the case of 1-jets one should operate with the Lie groups.

Now we specify our equivalence problem by a $G$-invariant differential equation $\mathfrak{N} \subset J_{r}^{l}(M)$ on submanifolds $N \subset M$ of codimension $r$. The symbol of this equation $h_{a}^{l} \subset S^{l} \mathfrak{t}_{a}^{*} \otimes \mathfrak{v}_{a}$ is a $\rho_{l, l-1}$-vertical subspace of $T_{a_{l}} \mathfrak{N}$. Since the pseudogroup $G$ acts on $\mathfrak{N}$, we obtain the following exact sequence:

$$
0 \rightarrow \mathfrak{h}_{a}^{l} \hookrightarrow \mathfrak{g}_{a}^{l} \stackrel{\lambda}{\longrightarrow} h_{a}^{l} \stackrel{\varpi}{\longrightarrow} \mathfrak{O}_{a}^{l} \rightarrow 0 .
$$

Definition 4. The quotient $\mathfrak{O}_{a}^{l}=h_{a}^{l} / \lambda\left(\mathfrak{g}_{a}^{l}\right)$ is called the space of $l$-covariants of the pseudogroup $G$ action. The dual $\left(\mathfrak{O}_{a}^{l}\right)^{*}$ is named the space of $l$-variants.

Our study of formal equivalence of submanifolds under the $G$-action is inductive and based on the following observation:

Proposition 2. Let $\left[N_{1}\right]_{a}^{l-1}=\left[N_{2}\right]_{a}^{l-1} \in \rho_{l, l-1}(\mathfrak{N})$ and $l>1$. The l-jets of submanifolds $N_{1}$ and $N_{2}$ from $\mathfrak{N}$ at a point $a \in M$ are $G$-equivalent if and only if they belong to the same $\mathfrak{g}_{a}^{l}$-orbit on $h_{a}^{l}$, which are are affine subspaces of codimension equal $\operatorname{dim} \mathfrak{O}_{a}^{l}=\operatorname{dim} h_{a}^{l}-\operatorname{dim}\left(\mathfrak{g}_{a}^{l} / \mathfrak{h} l a\right)$. In other words, this happens iff they have the same l-variants: $\varpi\left(\left[N_{2}\right]_{a}^{l}-\left[N_{1}\right]_{a}^{l}\right)=0$.

The requirement $l>1$ is related to remark 1 For $l=1$ there is difference between symbolic Lie groups and algebras: In the first case one gets orbits in the Grassmannian $J_{r}^{1}(M)=\mathrm{Gr}_{r}\left(T_{a} M\right)$, while in the latter one gets affine subspaces in its tangent space at $a_{1}$. Thus 1 -jets require a separate treatment.

\subsection{Differential invariants and Tresse derivatives}

Let $\mathcal{I}_{k}$ be the algebra of order $k$ differential invariants of the pseudogroup $G$ action on $\mathfrak{N}_{k}$ (the equation consists of pieces of different orders, see Appendix A.1), i.e. functions constant on the $G_{k}$-orbits in $\mathfrak{N}_{k}$. Denote by $\mathcal{I}$ the algebra of all differential invariants. It is filtered by the subalgebras $\mathcal{I}_{k}$ via the natural inclusion $\rho_{k+1, k}^{*}: \mathcal{I}_{k} \rightarrow \mathcal{I}_{k+1}$ if the pseudogroup $G$ is integrable. If the pseudogroup is not integrable, we can still consider its finite piece to order $l$. 
Sophus Lie proposed to produce new differential invariants via invariant differentiations $\nabla$. He suggested a theorem that a finite number of them $\nabla_{1}, \ldots, \nabla_{n}$ is enough to produce the whole algebra $\mathcal{I}$ from some $\mathcal{I}_{k}$.

An important case of invariant differentiations $\nabla_{i}: \mathcal{I}_{k} \rightarrow \mathcal{I}_{k+1}$ constitute derivatives a la Tresse, which we now introduce.

Suppose we have $n=\operatorname{dim} N=\operatorname{dim} M-r$ differential invariants $f_{1}, \ldots, f_{n}$ on $\mathfrak{N}_{k}$. Provided $\pi_{k+1, k}\left(\mathfrak{N}_{k+1}\right)=\mathfrak{N}_{k}$ we define the differential operator

$$
\hat{\partial}_{i}: C^{\infty}\left(\mathfrak{N}_{k}\right) \rightarrow \Omega^{1}\left(\mathfrak{N}_{k+1}^{\prime}\right),
$$

where $\mathfrak{N}_{k+1}^{\prime}$ is the open set of points $a_{k+1} \in \mathfrak{N}_{k+1}$ with

$$
\left.d f_{1} \wedge \ldots \wedge d f_{n}\right|_{L\left(a_{k+1}\right)} \neq 0 .
$$

We require that $\left\{f_{i}\right\}_{i=1}^{n}$ are such that $\mathfrak{N}_{k+1}^{\prime}$ is dense in $\mathfrak{N}_{k+1}$. For the trivial equation $\mathfrak{N}_{k+1}=J_{r}^{k+1}(M)$ this is always the case. But if the equation $\mathfrak{N}$ is proper, this is a requirement of "general position" for it. Given condition (5) we write:

$$
\left.d f\right|_{L\left(a_{k+1}\right)}=\left.\sum_{i=1}^{n} \hat{\partial}_{i}(f)\left(a_{k+1}\right) d f_{i}\right|_{L\left(a_{k+1}\right)}
$$

which defines the function $\hat{\partial}_{i}(f)$ uniquely at all the points $a_{k+1} \in \mathfrak{N}_{k+1}^{\prime}$. This yields an invariant differentiation $\hat{\partial}_{i}=\hat{\partial} / \hat{\partial} f_{i}: \mathcal{I}_{k} \rightarrow \mathcal{I}_{k+1}$. The expressions $\hat{\partial}_{i}(f)=\hat{\partial} f / \hat{\partial} f_{i}$ are called Tresse derivatives of $f$ with respect to $f_{i}$.

The above construction can be presented more effectively in a local chart $J^{k} \pi \subset J_{r}^{k}(M)$ (for this and the following notions we refer to Appendix A). Given a local submanifold $N \subset M$ we can find a transversal foliation of its neighborhood and locally identify it with a bundle $\pi$ over $N$. Then we can define Tresse derivative via the horizontal differential $\hat{d}: C^{\infty}\left(J^{k} \pi\right) \rightarrow \Omega^{1}\left(J^{k+1} \pi\right)$.

In coordinate language given 1-jet $a_{1}=[N]_{a}^{1}$ we choose local coordinates $\left(x^{i}, u^{j}\right)$ on $M$, with $\partial_{x^{i}}$ being tangent to $N$ at $a$ and $\partial_{u^{j}}$ being transversal. Then $\hat{d} f=\sum \mathcal{D}_{i}(f) d x^{i}$, where $\mathcal{D}_{i}$ is the operator of total derivative with respect to coordinate $x^{i}$.

In these terms condition (5) re-writes as:

$$
\hat{d} f_{1} \wedge \ldots \wedge \hat{d} f_{n} \neq 0 .
$$

(in an open set $U$ - a phrase we'll be omitting later on), i.e. the Jacobian $\left\|\mathcal{D}_{i}\left(f_{j}\right)\right\|$ is non-degenerate. Then for any other $f \in \mathcal{I}$ we have:

$$
\hat{d} f=\sum_{i} \hat{\partial}_{i}(f) \hat{d} f_{i}
$$

Thus

$$
\hat{d}=\sum d x^{i} \otimes \mathcal{D}_{x^{i}}=\sum \hat{d} f_{i} \otimes \hat{\partial} / \hat{\partial} f_{i},
$$

which yields the expression of Tresse derivatives:

$$
\hat{\partial}_{i} \stackrel{\text { def }}{=} \hat{\partial} / \hat{\partial} f_{i}=\sum_{j}\left(\mathcal{D}_{x^{a}}\left(f_{b}\right)\right)_{i j}^{-1} \mathcal{D}_{x^{j}},
$$


where $\left(\mathcal{D}_{x^{a}}\left(f_{b}\right)\right)$ is the Jacobian matrix in total derivatives. This formula can be interpreted as a "change of variables".

Informally speaking, $f_{i}$ are considered as base (horizontal) coordinates on the equation $\mathfrak{N}$. They are classically called differential parameters and in terms of them $\hat{\partial}_{i}$ are total derivatives. Then formula (6) has the standard sense.

This idea was realized by S. Lie for vertical actions. This means that the pseudogroup $G$ is represented in the equation $\mathfrak{N} \subset J^{l} \pi$ in such a way that every orbits in $\mathfrak{N}_{k}$ belongs to a $\pi_{k}$-fiber. The base functions $x^{1}, \ldots, x^{n}$ (for instance, local coordinates) are differential invariants. The corresponding Tresse derivative $\hat{\partial}_{i}$ coincides with the operator of total derivative $\mathcal{D}_{i}$ with respect to coordinate $x^{i}$.

Lie and his students believed this can fully extend to the general pseudogroup actions and Tresse seems to be the first who realized this.

\subsection{Covariants and equivalence}

We will present now an infinitesimal analog of the construction of differential invariants. Fix a point $a_{l} \in J_{r}^{l}(M)$ and define the increasing filtration of $T_{a_{l}}^{*} J_{r}^{l}(M)$ by

$$
\Theta_{k}\left(a_{l}\right)=\left\{d_{a_{l}} f \mid f \in \mathcal{I}_{k}\right\} \subset T_{a_{l}}^{*} J_{r}^{l}(M), \quad k=0, \ldots, l .
$$

Note that $\Theta_{l}$ is the 1 st order equation defining $G^{l}$-differential invariants on $J_{r}^{l}(M)$ at regular points. Near singular orbits the differential invariants have bad behavior, and there we define the filtration as follows (the definitions at regular points coincide):

$$
\Theta_{k}\left(a_{l}\right)=\pi_{l, k}^{*} \operatorname{Ann} T_{a_{k}}\left(G^{k} \cdot a_{k}\right) .
$$

Proposition 3. For $0<k \leq l$ : $\mathfrak{O}_{a}^{k}=\left(\Theta_{k} / \Theta_{k-1}\right)^{*}$.

Proof. In fact, $\mathfrak{O}_{a}^{k}=T_{a_{k}}\left(\pi_{k, k-1}\right)_{*}^{-1}\left(G^{k-1} \cdot a_{k-1}\right) / T_{a_{k}}\left(G^{k} \cdot a_{k}\right)$ and the claim follows.

Since $\left(\mathfrak{O}_{a}^{k}\right)^{*} \subset S^{k} \mathfrak{t}_{a} \otimes \mathfrak{v}_{a}^{*}$, we have the natural map

$$
\delta^{*}:\left(\mathfrak{O}_{a}^{k}\right)^{*} \otimes \mathfrak{t} \rightarrow\left(\mathfrak{D}_{a}^{k+1}\right)^{*},
$$

which can be viewed as the symbol of invariant differentiation at regular points. In order to prove surjectivity of this map for large $k$, we will investigate the dual map and prove its eventual injectivity, see $\$ 2.3$

If we have $n$ independent differential invariants of order $k$, then $\operatorname{dim} \mathfrak{O}^{k} \geq n$. In this case we can treat map (8) as an infinitesimal version of Tresse derivative. This will provide a finite set of generators for differential invariants, 2.4

Thus we get a solution to the formal equivalence problem by the following inductive procedure. We start with a pseudogroup $G$ and $\mathfrak{N}=J_{r}^{l}(M)$. Let the first nontrivial space of $l$-covariants be $\mathfrak{O}^{l}$. Fix $l$-variants from $\left(\mathfrak{O}_{a}^{l}\right)^{*}=\Theta^{l}$, 
i.e. fix order $l$ differential invariants. If they are compatible as differential operators (otherwise we need to add compatibility conditions), this yields a smaller equation $\mathfrak{N} \subset J_{r}^{l}(M)$ on submanifolds $N$ and we continue (in fact, the procedure is more complicated: If the invariants are not constants, we take some of them as "coordinates", express the others via them and fix the corresponding functions-relations). At regular points the procedure stops in a finite number of steps by the Cartan-Kuranishi prolongation theorem.

An important case is an eventual absence of $l$-variants.

Definition 5. (i) A pseudogroup $G$ is said to act l-transitively near $a_{l} \in \mathfrak{N}$, if for any other jet $b_{l} \in \mathfrak{N}$, close to $a_{l}$, there exists an element $\varphi_{l} \in G_{a, b}^{l}$ such that $\varphi_{l}\left(a_{l}\right)=b_{l}$. In other words, the orbit $G^{l} \cdot a_{l}$ is open.

(ii) An action of a pseudogroup $G$ is said to be l-transversal near $a_{l}$, if the above holds whenever $a_{l-1}=b_{l-1}$. In other words, $\mathfrak{G}_{a}^{l}$ acts transitively on $\mathfrak{F}\left(a_{l-1}\right)$.

To explain the word "transversality", consider the map $\lambda: \theta \mapsto \bar{\theta}$ from (2). The space $\lambda^{-1}\left(h_{a}^{l}\right) \subset S^{l} T_{a}^{*} M \otimes T_{a} M$ contains two subspaces $\mathrm{St}_{a}^{l}$ and $\mathfrak{g}_{a}^{l}$.

Let $l>1$. The following statement follows from (3), (4) and definitions:

Proposition 4. l-transversality of $G$ on $\mathfrak{N}$ is equivalent to any of the conditions:

- $\mathrm{St}_{a}^{l}$ is transversal to $\mathfrak{g}_{a}^{l}$ in $\lambda^{-1}\left(h_{a}^{l}\right)$ : $\mathrm{St}_{a}^{l}+\mathfrak{g}_{a}^{l}=\lambda^{-1}\left(h_{a}^{l}\right)$.

- There are no l-covariants: $\mathfrak{O}_{a}^{l}=0$.

$l$-transversality is an inductive step to get $l$-transitivity. Namely, we have:

Theorem 5. Let $G^{1} \cdot a_{1}$ be open and $G$ acts $j$-transversally near $a_{j}$ for $1<j \leq l$. Then $G$ acts l-transitively near $a_{l}$.

Definition 6. We will call an action of $G$ formally transitive if it is l-transitive near a generic point of $\mathfrak{N}$ for every $l$. If it is $l$-transversal for all $l$ starting from some $l_{0}$, we will call such an action eventually transitive. This basically means that the number of differential invariants is finite.

\section{Homological methods}

In this section we develop a technique to formally handle differential invariants and prove the finiteness theorem.

\subsection{Cohomology of covariants}

Consider a pseudogroup $G$ of order $k$. Denote

$$
\mathfrak{h}_{a}^{l-s, s}=\mathfrak{g}_{a}^{l-s} \otimes\left(\operatorname{Ann} \mathfrak{t}_{a} \wedge \Lambda^{s-1} T_{a}^{*} M\right)+\mathfrak{h}_{a}^{l-s} \otimes \Lambda^{s} T_{a}^{*} M
$$


and let $\varrho: T_{a}^{*} M \rightarrow \mathfrak{t}_{a}^{*}$ be the restriction map. Consider the following commutative diagram, where the horizontal arrows are induced $\delta$-differentials and the vertical ones are obvious from exact four-sequence (4).

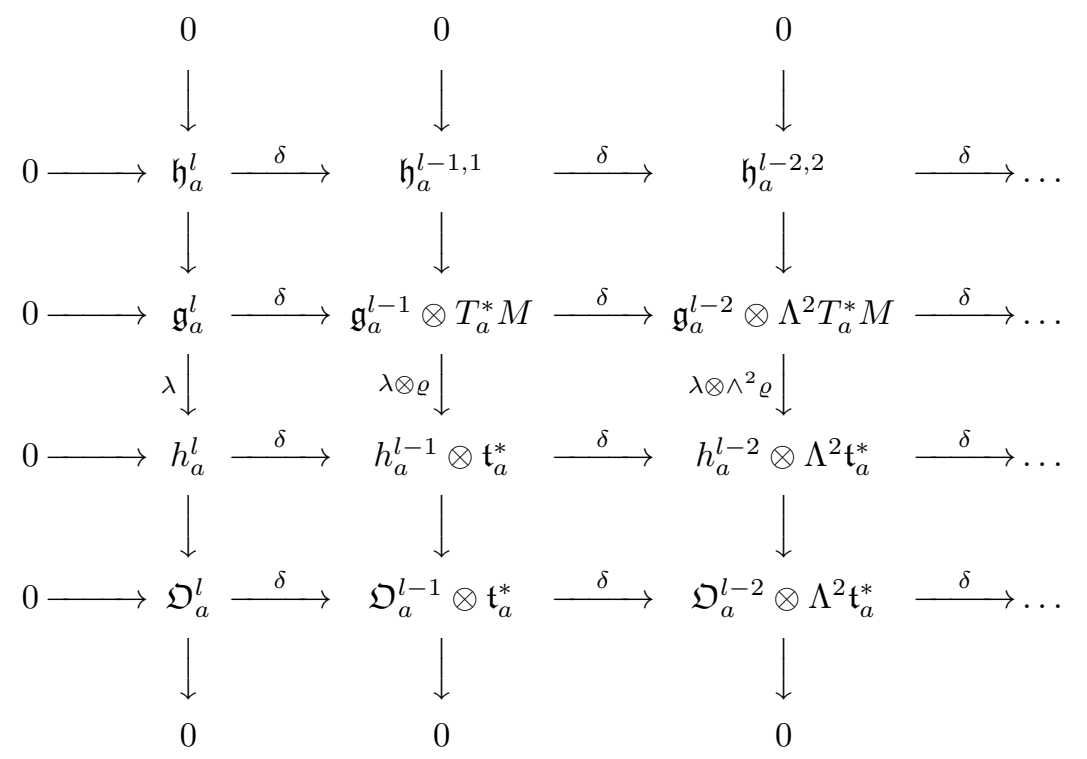

Denote the cohomology of the first line at the term $\mathfrak{h}^{l-s, s}$ by $H^{l-s, s}(\mathfrak{h}, \mathfrak{g})$ and the cohomology of the forth line at the term $\mathfrak{D}^{l-s} \otimes \Lambda^{s} \mathfrak{t}^{*}$ by $H^{l-s, s}(\mathfrak{O})$. These latter will be called the cohomology of covariants (in principle, they depend on the point of equation $\mathfrak{N}$, but we will not indicate this).

The following statement is obtained by the usual diagram chase.

Proposition 6. Suppose $H^{l-s-1, s+1}(\mathfrak{g})=H^{l-s-2, s+2}(\mathfrak{g})=0$ and $H^{l-s, s}(h)=$ $H^{l-s-1, s+1}(h)=0$. Then $H^{l-s, s}(\mathfrak{O}) \simeq H^{l-s-2, s+2}(\mathfrak{h}, \mathfrak{g})$.

Corollary 7. Let an order $k$ pseudogroup $G$ act on submanifolds $N \subset M$ of fixed codimension $r$, more precisely on $J_{r}^{k}(M)$. Let $G$ be $(q+2)$-acyclic and $l>k+1$. Then $H^{l-s, s}(\mathfrak{O}) \simeq H^{l-s-2, s+2}(\mathfrak{h}, \mathfrak{g})$ for all $s \leq \min (l-k-2, q)$. In particular, if $G$ is involutive, then the equality holds for all $s \leq l-k-2$.

Corollary 8. Consider a $(q+2)$-acyclic pseudogroup $G$ of order $k$ acting on an equation $\mathfrak{N} \subset J_{r}^{m}(M)$, which is $(p+1)$-acyclic. Let $l>\max (k+1, m)$. Then $H^{l-s, s}(\mathfrak{O}) \simeq H^{l-s-2, s+2}(\mathfrak{h}, \mathfrak{g})$ for $s \leq \min (l-k-2, l-m-1, p, q)$.

Corollary 9. Suppose that: 1) $\mathfrak{O}_{a}^{l-1}=0$; 2) $h_{a}^{l}=\left(h_{a}^{l-1}\right)^{(1)}, \mathfrak{g}_{a}^{l}=\left(\mathfrak{g}_{a}^{l-1}\right)^{(1)}$; 3) $H^{l-2,2}\left(\mathfrak{g}_{a}\right)=0$. Then $\mathfrak{O}_{a}^{l}=H^{l-2,2}\left(\mathfrak{h}_{a}, \mathfrak{g}_{a}\right)$.

Thus we obtain a method to calculate recursively the space of covariants $\mathfrak{O}_{a}^{l}$ if we know the cohomology groups $H^{*, *}(\mathfrak{h}, \mathfrak{g})$. This leads to the inductive approach of 1.4 to the equivalence problem. Due to proposition 6

$$
H^{l, 0}(\mathfrak{h}, \mathfrak{g})=0, \quad H^{l, 1}(\mathfrak{h}, \mathfrak{g})=0 \text { for } l \geq k .
$$


Let us calculate the groups $H^{l, s}(\mathfrak{h}, \mathfrak{g})$ for $s>1$. We do it at first with an additional assumption of non-characteristisity.

Theorem 10. Let $G$ be $q$-acyclic and let $c=\min (l-k, q)$. Denote by $H^{l-s, s}(\mathfrak{h})$ the cohomology group of the complex

$$
0 \rightarrow \mathfrak{h}_{a}^{l} \rightarrow \mathfrak{h}_{a}^{l-1} \otimes \mathfrak{t}_{a}^{*} \rightarrow \mathfrak{h}_{a}^{l-2} \otimes \Lambda^{2} \mathfrak{t}_{a}^{*} \rightarrow \ldots
$$

at the term $\mathfrak{h}_{a}^{l-s} \otimes \Lambda^{s} \mathfrak{t}_{a}^{*}$.

Suppose that the subspace $\mathfrak{t}_{a} \subset T_{a} M$ is strongly non-characteristic for $\mathfrak{g}_{a}$ [KL3], i.e. $\operatorname{Ann}\left(\mathfrak{t}_{a}\right) \circ S^{k-1} T_{a}^{*} M \otimes T_{a} M \cap \mathfrak{g}_{a}=0$. Then for $0 \leq s<c$ we have:

$$
H^{l, s}(\mathfrak{h}, \mathfrak{g})=H^{l, s}(\mathfrak{h}) .
$$

Proof. Consider the following commutative diagram of vertical exact threesequences, where $\delta^{\prime}$ is the induced differential:

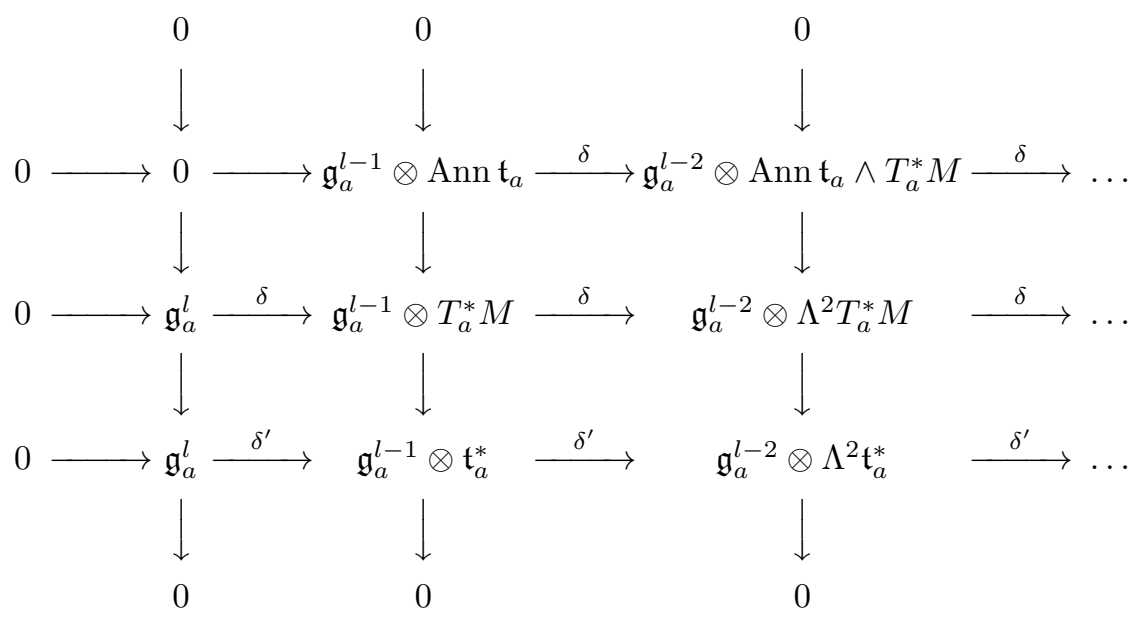

The middle line is $c$-acyclic. If $\mathfrak{t}_{a}$ is strongly non-characteristic, we have the same property for the bottom line [KL3. Let $H^{i, j}(\mathfrak{g} \otimes \mathrm{Ann} \mathfrak{t} ; \delta)$ denote the cohomology of the first complex at the term $\mathfrak{g}_{a}^{i} \otimes \operatorname{Ann} \mathfrak{t}_{a} \wedge \Lambda^{j} T_{a}^{*} M$. A diagram chase gives: $H^{i-1, j}(\mathfrak{g} \otimes$ Ann $\mathfrak{t} ; \delta) \simeq H^{i, j}\left(\mathfrak{g}, \delta^{\prime}\right)=0$ for $i>k, 0 \leq j<c$.

Consider the following commutative diagram with vertical three-sequences being exact. Note that if the subspace $\mathfrak{t}_{a}$ is strongly non-characteristic, we can consider $\mathfrak{h}_{a}^{l} \subset S^{l} \mathfrak{t}_{a}^{*} \otimes \mathfrak{t}_{a}$, so that the bottom complex is the usual Spencer 
$\delta$-complex on $\mathfrak{t}_{a}$.

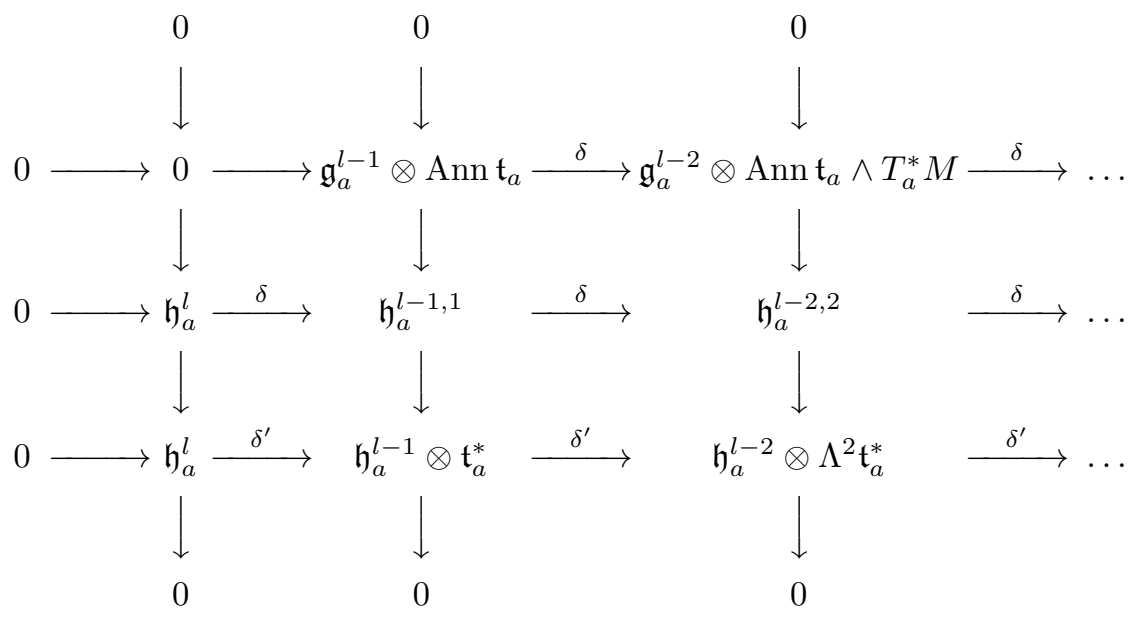

Since the first horizontal complex is $c$-acyclic, the middle and the bottom complexes have the same cohomology in the first $c$ terms.

In the Spencer complex on $\mathfrak{t}_{a}$ all $\delta$-cohomology groups eventually vanish (Poincaré $\delta$-lemma [S] [KLV]). In non-characteristic case for a big number $i$ (actually such big that the equation $G$ on $T M$ and its restriction to $\mathfrak{t}$ as well as the equation $\mathfrak{N}$ on $\mathfrak{t}$ become involutive) we have: $H^{i, j}(\mathfrak{O})=0$.

Thus if the pseudogroup does not have all subspaces of given codimension $r$ weakly characteristic KL3, then we have the following finiteness theorem: Cohomology of covariants eventually vanish (on an open dense subset of the equation $\mathfrak{N}$ ). We will prove in 2.3 that this is a general fact.

Note however that with the approach of this section we calculated the cohomology of covariants, which is an important invariant of pseudogroup action:

Corollary 11. Suppose that assumptions of Proposition $\square$ and Theorem 10 hold. Then $H^{l-s, s}(\mathfrak{O}) \simeq H^{l-s-2, s+2}(\mathfrak{h})$ for $0 \leq s<c$.

\subsection{Criterion of transversality}

By Corollary 9 a very important cohomology group of $\mathfrak{h}$ is $H^{l, 2}(\mathfrak{h})$.

Theorem 12. Let a pseudogroup $G$ be 2-acyclic: $H^{l, 2}(G)=0, l \geq k$. Suppose that for some number $l_{0}>k$ the submanifold $N$ at a point a is $l_{0}$-transversal with respect to the pseudogroup $G$ action. Assume also that $H^{l, 2}(\mathfrak{h})=0$ and $H^{l, 1}\left(\mathfrak{g}, \delta^{\prime}\right)=0$ for $l>l_{0}$. Then $N$ is l-transversal for all $l>l_{0}$ at $a$.

Proof. Indeed, from the first diagram of the proof of Theorem 10 we get: $H^{l-1,1}(\mathfrak{g} \otimes \operatorname{Ann} \mathfrak{t} ; \delta) \simeq H^{l, 1}\left(\mathfrak{g}, \delta^{\prime}\right)=0$.

From the second diagram of the same proof we obtain that since $H^{l-1,1}(\mathfrak{g} \otimes$ Ann $\mathfrak{t} ; \delta)=0$ the map of cohomology $H^{l-2,2}(\mathfrak{h} ; \mathfrak{g}) \rightarrow H^{l-2,2}(\mathfrak{h})$ is injective. Thus by assumptions and corollary $9 \mathfrak{O}_{a}^{l} \simeq H^{l-2,2}\left(\mathfrak{h}_{a} ; \mathfrak{g}_{a}\right)=0$ for all $l \leq l_{0}$. 
Corollary 13. With the assumptions of Theorem 12 the pseudogroup action is eventually transitive. If we assume in addition that $N$ is l-transversal with respect to the pseudogroup $G$ action for all $l<l_{0}$ and that the orbit $G^{1} \cdot a_{1}$ is open, then the action of $G$ is formally transitive around a.

Note that in the theorem we don't require $\mathfrak{t}_{a}$ to be strongly non-characteristic. This means that zero cohomology of the bottom complexes from diagrams in Theorem 10 can be non-vanishing even for large $l$.

However often the other cohomology groups vanish in stable range (big $l$ ). This is related to the following fact:

Proposition 14. Let the pseudogroup $G$ be 2-acyclic from some level $l_{0}$. Suppose that $H^{l, 1}\left(\mathfrak{g}_{a}, \delta^{\prime}\right)=0$ for $l \geq l_{0}$. Then $H^{l, 1}\left(\mathfrak{h}_{a}\right)=0$.

Proof. From the first diagram of Theorem [10 we get the isomorphism $H^{l-1,1}(\mathfrak{g} \otimes$ Ann $\mathfrak{t} ; \delta) \simeq H^{l, 1}\left(\mathfrak{g} ; \delta^{\prime}\right)=0$.

From the second diagram since $H^{l-1,1}(\mathfrak{g} \otimes$ Ann $\mathfrak{t} ; \delta)=0$ we obtain that the map of cohomology $H^{l, 1}(\mathfrak{h} ; \mathfrak{g}) \rightarrow H^{l, 1}(\mathfrak{h})$ is surjective. The claim follows from the fact that $H^{l, 1}(\mathfrak{h} ; \mathfrak{g})=0$.

This means that the complex (9) is natural in the following sense:

$$
\mathfrak{h}^{l}=\left\{\theta \in \mathfrak{g}^{l} \mid \partial_{v}(\theta) \in \mathfrak{h}^{l-1} \forall v \in \mathfrak{t}_{a}\right\} .
$$

Notice that there exists an important necessary condition for eventual (and hence formal) transitivity of the pseudogroup action. This is a purely dimensional obstruction to transversality.

Namely, by proposition 4 -transversality condition imposes the following inequality on the symbol $h_{a}^{l}$ of the equation $\mathfrak{N}$ :

$$
\operatorname{dim} \mathfrak{g}_{a}^{l} \geq \operatorname{dim} h_{a}^{l} .
$$

This easy-to-check condition is often helpful. Namely, in many cases its fulfilment implies transversality for generic submanifolds $N$ (see examples below).

\subsection{Finiteness theorem}

Here we prove an algebraic point-wise version of the finiteness theorem. Its local version will appear in the next section.

For a Lie pseudogroup $G$ the corresponding Lie equation for vector fields is

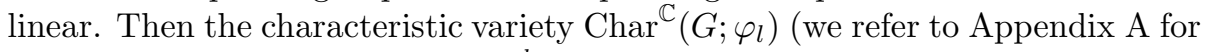
the definition and properties) of $G^{l}$ depends only on the base point $a=\rho_{l, 0}\left(\varphi_{l}\right)$.

More generally, the same holds for any pseudogroup $G$ after some number of prolongations, i.e. for some $l \geq l_{0}$. Indeed, if $G^{l}$ has prolongation over points $\varphi_{l}^{\prime}, \varphi_{l}^{\prime \prime} \in \rho_{l, 0}^{-1}(a)$ (this is given by the conditions $W_{j}\left(G ; \varphi_{j}^{\prime}\right)=W_{j}\left(G ; \varphi_{j}^{\prime \prime}\right)=0$ ), then the characteristic varieties $\operatorname{Char}^{\mathbb{C}}\left(G ; \varphi_{j}^{\prime}\right)$, $\operatorname{Char}^{\mathbb{C}}\left(G ; \varphi_{j}^{\prime \prime}\right) \subset P^{\mathbb{C}} T_{a}^{*} M$ coincide. We will denote the characteristic variety also by $\operatorname{Char}^{\mathbb{C}}\left(\mathfrak{g}_{a}\right)$. 
Theorem 15. The Grassmannian space $J_{r}^{1}(M)_{a}=\operatorname{Gr}_{n}\left(T_{a} M\right)$ of n-dimensional subspaces $\mathfrak{t}_{a} \subset T_{a} M, n+r=m=\operatorname{dim} M$, contains an open dense subset $\mathcal{U}_{G}(a)$, depending only on $\mathrm{Char}^{\mathbb{C}}\left(\mathfrak{g}_{a}\right)$, and there exists a number $l_{0}$, depending only on the pseudogroup $G$ and the equation for submanifolds $\mathfrak{N}$, such that the following holds. For any point $a_{l} \in \mathfrak{N}_{l}, l \geq l_{0}$, with $a=\rho_{l, 0}\left(a_{l}\right)$ and such that $a_{1}=\rho_{l, 1}\left(a_{l}\right)$ is an admissible tangent space $\mathfrak{t}_{a} \in \mathcal{U}_{G}(a)$ we have:

$$
H^{i, j}(\mathfrak{O})=0 \quad \text { for any } i+j=l \geq l_{0} .
$$

Proof. The proof of the theorem is split into two two parts, depending on weather $r \leq \operatorname{codim} \operatorname{Char}^{\mathbb{C}}(\mathfrak{g})$ or $r \geq \operatorname{codim} \operatorname{Char}^{\mathbb{C}}(\mathfrak{g})$ (in the case of equality both approaches are equivalent).

Note that the codimension does not depend on weather we consider affine variety in $T_{x}^{\mathbb{C}} M$ or its projectivization in $P T_{x}^{\mathbb{C}} M$ ( $P$ denotes projectivization and $\mathbb{C}^{\mathbb{C}}$ - complexification). However usage of complex characteristics is crucial. Also note that we do not require the characteristic variety to be irreducible, but take $d=\operatorname{codim} \operatorname{Char}^{\mathbb{C}}(\mathfrak{g})$ to be the codimension of its regular component (so this value is the minimum of codimensions by all regular points of all irreducible pieces). We have: $d \in[0, n]$.

1. $r \leq d$. In this case $\mathcal{U}_{G}(a)$ consists of subspaces $\mathfrak{t}$ such that $P \operatorname{Ann}(\mathfrak{t})^{\mathbb{C}}$ does not intersect $\operatorname{Char}^{\mathbb{C}}(\mathfrak{g})$. It is possible by Noether normalization lemma and all generic subspaces $\mathfrak{t}$ are such.

For a vector space $V$ denote by $S V=\oplus S^{i} V$ the ring of homogeneous polynomials on $V^{*}$. Let $I_{0}(\mathfrak{g})$ be the annihilator of the subvariety $\operatorname{Char}^{\mathbb{C}}(\mathfrak{g}) \subset$ $P\left(T_{a}^{*} M\right)^{\mathbb{C}}$, i.e. the ideal of homogeneous polynomials vanishing on the characteristic variety. It equals the radical of the characteristic ideal $I(\mathfrak{g}) \subset S\left(T_{a}^{\mathbb{C}} M\right)$ (see Appendix A); here again $S\left(T_{a}^{\mathbb{C}} M\right)=\oplus S^{i}\left(T_{a}^{\mathbb{C}} M\right)$ is the polynomial algebra.

In addition Noether lemma states [M] that the projection along annihilator $P_{\mathfrak{t}}:$ Char $^{\mathbb{C}}(\mathfrak{g}) \rightarrow P\left(\mathfrak{t}^{*}\right)^{\mathbb{C}}$ is a finite-to-one closed map such that the homogeneous ring $S\left(T_{a}^{\mathbb{C}} M\right) / I_{0}(\mathfrak{g})$ is a finitely generated module over the algebra $S\left(\mathfrak{t}^{\mathbb{C}}\right)$.

We claim that the homogeneous ring $S\left(T_{a}^{\mathbb{C}} M\right) / I(\mathfrak{g})$ is a finitely generated module over the algebra $S\left(\mathfrak{t}^{\mathbb{C}}\right)$. Indeed, let us have a polynomial relation in the $\operatorname{ring} S\left(T_{a}^{\mathbb{C}} M\right) / I_{0}(\mathfrak{g})$ :

$$
Q\left(f_{1}, \ldots, f_{m}\right) \in I_{0}(\mathfrak{g}), \quad f_{i} \in S\left(T_{a}^{\mathbb{C}} M\right), Q \in S\left(\mathfrak{t}^{\mathbb{C}}\right) .
$$

Denote by $N$ the minimal integer number such that $I_{0}(\mathfrak{g})^{N} \subset I(\mathfrak{g})$. Then we have the following polynomial relation in the $\operatorname{ring} S\left(T_{a}^{\mathbb{C}} M\right) / I(\mathfrak{g})$ :

$$
Q^{N}\left(f_{1}, \ldots, f_{m}\right) \in I(\mathfrak{g}) .
$$

Thus the characteristic module $\mathfrak{g}^{*}$, dual to the symbolic system $\mathfrak{g}$ (see Appendix $[$, is Noetherian over $S(\mathfrak{t})$ (informally: the symbolic module grows over the characteristic variety and it is projected finite-to-one). Thus the Koszul cohomology of $\mathfrak{g}^{*}$ is finite. Dualization yields finiteness of the Spencer cohomology $H^{*, *}\left(\mathfrak{g}, \delta^{\prime}\right)$ of $\mathfrak{g}$ over $\mathfrak{t}$. 
Alternatively the latter claim follows from Poincar'e $\delta$-lemma [S, KLV]. The bound $l_{0}$ such that $H^{i, j}\left(\mathfrak{g}, \delta^{\prime}\right)=0$ for $i \geq l_{0}$ depends only on dimensions of the module $\mathfrak{g}^{*}$ over the algebra $S(\mathfrak{t})$ and so is universal over all $\mathfrak{t} \in \mathcal{U}_{G}(a)$.

Let us take $l_{0}$ such that the $l^{\text {th }}$ Spencer $\delta$-complexes for $\mathfrak{g}$ and $h$ are acyclic, when $l \geq l_{0}$. For such $l$ in the first commutative diagram from the proof of Theorem 10 the second and the third complexes are acyclic. Therefore the first one is acyclic. It is also the first complex of the second diagram from the proof, so that we get isomorphism between the cohomology of the second and third complexes, i.e.

$$
H^{i, j}(\mathfrak{h}, \mathfrak{g}) \simeq H^{i, j}(\mathfrak{h}) \quad \text { for } i+j=l \geq l_{0} .
$$

From the above isomorphism we deduce vanishing of the zero and first cohomology of complex (9) in the range $l \geq l_{0}$. This means that for these $l$ the space $\mathfrak{h}^{l+1}$ is the Spencer $\delta$-prolongation of the space $\mathfrak{h}^{l}$. This implies (again by Poincaré $\delta$-lemma) that the cohomology $H^{i, j}(\mathfrak{h})$ vanish for big $i+j=l$. Thus increasing $l_{0}$ properly, we obtain that the cohomology $H^{i, j}(\mathfrak{h}, \mathfrak{g})=0$ for $i+j=l \geq l_{0}$.

Now the claim follows from Proposition 6 because the cohomology of covariants coincide with the cohomology $H^{*, *}(\mathfrak{h}, \mathfrak{g})$ in the stable range $l \geq l_{0}$.

2. $r \geq d$. In this case $\mathcal{U}_{G}(a)$ consists of subspaces $\mathfrak{t}$ such that the projection of $\operatorname{Char}^{\mathbb{C}}(\mathfrak{g})$ along $P \operatorname{Ann}(\mathfrak{t})^{\mathbb{C}}$ on $P\left(\mathfrak{t}^{*}\right)^{\mathbb{C}}$ is surjective. Again all generic subspaces $\mathfrak{t}$ are such due to Noether normalization lemma $\underline{M}$.

An element $v \in T_{a} M$ is regular (in the sense of commutative algebra $\mathrm{AB}$, BH] ) if it does not belong to the annihilator $I(\mathfrak{g})$ of the module $\mathfrak{g}^{*}$. This means that $P \operatorname{Ann}(v)^{\mathbb{C}}$ does not contain the characteristic variety $\operatorname{Char}^{\mathbb{C}}(\mathfrak{g})$. This is equivalent to the fact that the projection of $\operatorname{Char}^{\mathbb{C}}(\mathfrak{g})$ along $P \operatorname{Ann}(v)^{\mathbb{C}}$ to $P(\mathbb{C} v)^{*}$ is not empty and is therefore surjective.

More generally, a sequence $\left(v_{1}, \ldots, v_{n}\right)$ is regular $\left(\mathfrak{g}^{*}\right.$-sequence) iff the projection of $\operatorname{Char}^{\mathbb{C}}(\mathfrak{g})$ along $P \operatorname{Ann}\left(v_{1}, \ldots, v_{n}\right)^{\mathbb{C}}$ to $P\left(\left\langle v_{1}, \ldots, v_{n}\right\rangle^{\mathbb{C}}\right)^{*}$ is surjective. We conclude that there exists a regular sequence $\left(v_{1}, \ldots, v_{n}\right)$ in $\mathfrak{t}$ of length $n=m-r=\operatorname{dim} \mathfrak{t} \leq m-d$.

This implies that all the Koszul homology of the module $\mathfrak{g}^{*}$ w.r.t. the sequence $\left(v_{1}, \ldots, v_{n}\right)$, or equivalently with coefficients in $\mathfrak{t}$, vanish except for the zero cohomology group, see $\mathrm{AB}$. or the appendix (including a letter of Serre) in GS1 (equivalently we can say that $\mathfrak{g}^{*}$ is a Cohen-Macaulay module over $S(\mathfrak{t}$ ), which implies the same result $\mathrm{AB},(\mathrm{BH})$ ). Dualizing this statement we obtain that the Spencer cohomology groups vanish: $H^{i, j}\left(\mathfrak{g}, \delta^{\prime}\right)=0, i \geq k, 0<j \leq n$. The zero cohomology group $H^{i, 0}\left(\mathfrak{g}, \delta^{\prime}\right)$ for $r>d$ is always non-zero and can be non-zero even for $r=d$.

As in the first case we use two diagrams from Theorem 10 to conclude that the second and the third complexes of the second diagram have the same cohomology, save for the zero cohomology (which is zero for the second complex, but can be non-zero for the third one):

$$
H^{i, j}(\mathfrak{h}, \mathfrak{g})=H^{i, j}(\mathfrak{h}) \quad \text { for } i \geq k, j>0 .
$$


In particular, $H^{i, 1}(\mathfrak{h})=0$ for $i \geq k$. This again yields that the positive cohomology of $\mathfrak{h}$ eventually vanish: $H^{i, j}(\mathfrak{h})=0$ for $i+j=l \geq l_{0}, j>0$. Consequently, $H^{i, j}(\mathfrak{h}, \mathfrak{g})=0$ for $i+j=l \geq l_{0}$ and all $j$.

Applying Proposition [ 6e again get vanishing of the cohomology of covariants $H^{i, j}(\mathfrak{O})$ in the stable range $i+j=l \geq l_{0}$.

Notice that with the approach of Theorem [15] the estimate for the place, where cohomology vanish, can be much higher than that one of Theorem 10. However the latter case works only for pseudogroups such that not all subspaces are weakly characteristic. But for some important pseudogroups, like volumepreserving or symplectic pseudogroups, all tangent subspaces are weakly characteristic. The finiteness theorem however still holds even in such cases.

Remark 2. There is another approach to prove Theorem [15. Namely, for big $l_{0}$ the symbolic system $\left\{\mathfrak{g}^{l}\right\}_{l \geq l_{0}}$ is involutive, so that all Spencer $\delta$-cohomology groups vanish. This means that $S\left(T_{a} M\right)$-module $\oplus_{l} \geq l_{0}\left(\mathfrak{g}^{l}\right)^{*}$ is Cohen-Macaulay. Then almost every subspace $\mathfrak{t}$ contains a regular sequence and then its positive Koszul homology vanish, so that $H^{i, j}\left(\mathfrak{g}, \delta^{\prime}\right)=0$ for $i \geq l_{0}$ and $j>0$.

With this approach we however cannot explicitly formulate which subspaces $\mathfrak{t}$ are good for vanishing of positive cohomology of the complex

$$
0 \rightarrow \mathfrak{g}_{a}^{l} \stackrel{\delta^{\prime}}{\longrightarrow} \mathfrak{g}_{a}^{l-1} \otimes \mathfrak{t}_{a}^{*} \stackrel{\delta^{\prime}}{\longrightarrow} \mathfrak{g}_{a}^{l-2} \otimes \Lambda^{2} \mathfrak{t}_{a}^{*} \rightarrow \cdots .
$$

This is the crucial place in the proof and the rest is just the diagram chase.

\subsection{Relation to the theorems of Lie and Tresse}

Let us formulate now the regularity assumptions. We let the point $a_{l}$ vary over $\mathfrak{N}_{l}$ with big enough $l \geq l_{0}$, so that the ranks of the symbol bundles are locally constant, $\delta$-cohomology are stabilized etc.

We call a point $a_{l}$ regular if the space $\mathfrak{t}_{a}=a_{1}=\rho_{l, 1}\left(a_{l}\right)$ is admissible in the sense of Theorem 15. The collection of such points is open and will be denoted by:

$$
\operatorname{Reg}_{l}^{1}(\mathfrak{N}, G)=\left\{a_{l} \in \mathfrak{N}_{l} \mid a_{1} \in \mathcal{U}_{G}(a)\right\} .
$$

We want to claim that $\operatorname{Reg}_{l_{0}}^{1}(\mathfrak{N}, G)$ is dense in $\mathfrak{N}_{l_{0}}$. This is so if the equation $\mathfrak{N}$ is trivial - defined by empty set of relations, i.e. $\mathfrak{N}_{l}=J_{r}^{l}(M)$.

More generally, each equation $\mathfrak{N}$ with sufficiently rich $\mathfrak{N}_{1}$ is such, meaning that any jet $a_{1}$ can be perturbed to $a_{1}^{\prime}$ in the fiber over $a=\rho_{1,0}\left(a_{1}\right)$ to satisfy the transversality conditions of Theoren[15] $a_{1}^{\prime} \in \mathcal{U}_{G}(a)$. In other words, $\mathfrak{N}_{1}$ is not contained in the $G$-invariant singular equation $\cup_{a \in M}\left[\operatorname{Gr}_{n}\left(T_{a} M\right) \backslash \mathcal{U}_{G}(a)\right]$.

We will need another assumption, which is similar to Kumpera's hypothesis $\mathrm{H}_{3}$ Kum. Denote by $\Delta_{l}\left(a_{l}\right)$ the tangent space to the $G_{l}$-orbit through $a_{l} \in \mathfrak{N}_{l}$. Recall that each $a_{l+1} \in \mathfrak{N}_{l}^{(1)}$ determines a horizontal space $L\left(a_{l+1}\right) \subset T_{a_{l}} \mathfrak{N}_{l}$. Consider the open set

$$
\operatorname{Reg}_{l}^{2}(\mathfrak{N}, G)=\left\{a_{l} \in \mathfrak{N}_{l} \mid \exists a_{l+1} \in \mathfrak{N}_{l+1}: \Delta_{l}\left(a_{l}\right) \cap L\left(a_{l+1}\right)=0\right\} .
$$


We want to claim that $\operatorname{Reg}_{l_{0}}^{2}(\mathfrak{N}, G)$ is also dense. This can fail, for instance, if we have $l$-transversality. For the trivial equation $\mathfrak{N}_{l}=J_{r}^{l}(M)$ the condition means that we have $n$ independent differential invariants $f_{1}, \ldots, f_{n}$. However for proper $\mathfrak{N}$ this is a requirement on the equation.

Given these two regularities we will prove that the algebra of differential invariants has a finite base w.r.t. Tresse derivatives (on an open dense set; but this condition is natural since usually the differential invariants have singularities).

S. Lie used invariant differentiations to generate differential invariants. A. Tresse observed that they can be obtained if we have a sufficient number of independent differential invariants.

Remark 3. A. Kumpera proved Lie-Tresse theorem for a Lie sheaf of vector fields [Kum]. Under his conditions the maps $\lambda \otimes \Lambda \varrho$ in the four-line diagram of $\$ 2.1$ are injective, so that the first complex of it vanishes. Then the diagram becomes with 3-lines and after regularity assumptions the vanishing theorem follows from the stabilization of the cohomology of pseudogroup $G$ as CartanKuranishi theorem [Kur] states.

We will deduce now the theorem of Lie-Tresse. We will assume at first that both equations $G$ of the pseudogroup and $\mathfrak{N}$ for the submanifolds are formally integrable.

Theorem 16. Let a pseudogroup $G$ act on an equation $\mathfrak{N}$. Suppose that both are formally integrable. Let also $\operatorname{Reg}_{l_{0}}^{1}(\mathfrak{N}, G)$ and $\operatorname{Reg}_{l_{0}}^{2}(\mathfrak{N}, G)$ be dense in $\mathfrak{N}_{l_{0}}$. Then the infinitely prolonged equation $\mathfrak{N}^{(\infty)}$ contains a (no more than countable) collection of open $G$-invariant sets $U_{\alpha}$, the union of which $U=\cup_{\alpha} U_{\alpha}$ is dense, with the following properties.

Consider some $U_{\alpha}$. Then there are $n$ differential invariants $f_{1}, \ldots, f_{n}$ on it, with the corresponding invariant differentiations $\hat{\partial}_{1}, \ldots, \hat{\partial}_{n}$, and some other differential invariants $g_{1}, \ldots, g_{m}$ such that all differential invariants in $U_{\alpha}$ can be expressed via the $g_{j}$ and their invariant derivatives $\hat{\partial}^{J}\left(g_{j}\right)$ (for a multi-index $J=\left(j_{1}, \ldots, j_{n}\right)$ we denote $\left.\hat{\partial}^{J}=\hat{\partial}_{1}^{j_{1}} \ldots \hat{\partial}_{n}^{j_{n}}\right)$.

Usually (so-called regularity assumptions) there is only one such set $U$.

Proof. By the assumption $U\left(l_{0}\right)=\operatorname{Reg}_{l_{0}}^{1}(\mathfrak{N}, G) \cap \operatorname{Reg}_{l_{0}}^{2}(\mathfrak{N}, G)$ is dense in $\mathfrak{N}_{l_{0}}$. If $\mathfrak{N}_{l}=\mathfrak{N}_{l_{0}}^{\left(l-l_{0}\right)}$, then the characteristic variety on the level $l$ is the same as on the level $l_{0}$. Thus $\operatorname{Reg}_{l}^{1}=\rho_{l, l_{0}}^{-1}\left(\operatorname{Reg}_{l_{0}}^{1}\right) \cap \mathfrak{N}_{l}$. The same applies to $\operatorname{Reg}_{l}^{2}$. Thus we let $U=\rho_{\infty, l_{0}}^{-1}\left(U\left(l_{0}\right)\right)$ and this set can be represented as a union of sets $U_{\alpha}=\rho_{\infty, l_{0}}^{-1}\left(U_{\alpha}\left(l_{0}\right)\right)$.

Without loss of generality we suppose that each $U_{\alpha}$ belongs to a local chart, so that we can work with the jets of a bundle $J^{l} \pi$, which has a convenient representation (7). Moreover since $U_{\alpha}(l) \subset \operatorname{Reg}_{l}^{2}(\mathfrak{N}, G)$ for $l \geq l_{0}$ there are $n=\operatorname{dim} \mathfrak{t}$ differential invariants $f_{1}, \ldots, f_{n}$ such that $\hat{d} f_{1} \wedge \ldots \wedge \hat{d} f_{n} \neq 0$ (we can shrink $U_{\alpha}$ ). Thus the Tresse derivatives $\hat{\partial}_{i}=\hat{\partial} / \hat{\partial} f_{i}$ are well-defined in $U_{\alpha}$.

Let us calculate the symbols of these differentiations. For this we need a lift of vectors to invariant differentiations, described below. 
Consider a jet $a_{l+1} \in \mathfrak{N}_{l+1}, a_{l}=\pi_{l+1, l}\left(a_{l+1}\right), l \geq l_{0}$. Restrictions of $\hat{d} f_{i}$ to the horizontal plane $L\left(a_{l+1}\right) \subset T_{a_{l}} \mathfrak{N}_{l}$ form a basis. Thus we have a basis $e_{1}^{*}, \ldots, e_{n}^{*}$ of $\mathfrak{t}_{a}^{*}$ given by $\pi_{l}^{*} e_{i}^{*}=\left.\hat{d} f_{i}\right|_{L\left(a_{l+1}\right)}$.

Denote by $e_{1}, \ldots, e_{n}$ the dual basis of $\mathfrak{t}_{a}$ and let $v \in \mathfrak{t}_{a}$. Decompose $v=$ $\sum_{i=1}^{n} v_{i} e_{i}$. Choose a system of local coordinates $\left(x^{i}\right)_{i=1}^{n}$ near $a \in M$ such that $e_{i}=\partial_{x^{i}}$ at $a$. Then the symbol map

$$
\left(\mathfrak{D}_{a}^{l}\right)^{*} \otimes \mathfrak{t}_{a} \longrightarrow\left(\mathfrak{D}_{a}^{l+1}\right)^{*}
$$

is given by the formula

$$
\left[d_{a_{l}} f\right] \otimes v \mapsto\left[\sum_{i=1}^{n} v_{i} \mathcal{D}_{x^{i}}(f)\right] .
$$

Here $\left[d_{a_{l}} f\right]$ represents an element of $\left(\mathfrak{O}_{a}^{l}\right)^{*} \subset S^{l} \mathfrak{t}_{a} \otimes \mathfrak{v}_{a}^{*}$ and in the right-hand-side we restrict the covector at the point $a_{l+1}$ to the vertical subspace $T_{a_{l+1}}^{\mathrm{vert}} \mathfrak{N}_{l+1} \subset$ $S^{l+1} \mathfrak{t}_{a}^{*} \otimes \mathfrak{v}_{a}$ and take the quotient. The result does not depend on the coordinate system $\left(x^{i}\right)_{i=1}^{n}$ adapted at $a$ as indicated above.

Recall that $\left(\mathfrak{O}_{a}^{l+1}\right)^{*}=\left\{d f \mid f \in \mathcal{I}_{l+1}\right\} /\left\{d f \mid f \in \mathcal{I}_{l}\right\}$. Since $l \geq l_{0}$ and $U_{\alpha}(l) \subset$ $\operatorname{Reg}_{l}^{1}(\mathfrak{N}, G)$, the cohomology of covariants vanish, which means that map (11) is epimorphic. This implies that

$$
\mathcal{I}_{l+1}=\left\langle\rho_{l+1, l}^{*}\left(\mathcal{I}_{l}\right), \hat{\partial}_{1}\left(\mathcal{I}_{l}\right), \ldots, \hat{\partial}_{n}\left(\mathcal{I}_{l}\right)\right\rangle .
$$

Indeed, by the finiteness theorem the differentials of the functions on the right span the whole space of differentials of the functions to the left. The claim follows from the implicit function theorem.

Remark 4. We explained the density condition of $\operatorname{Reg}_{l_{0}}^{1}(\mathfrak{N}, G)$ before Remark 3. In certain cases density of $\operatorname{Reg}_{l_{0}}^{2}(\mathfrak{N}, G)$ in $\mathfrak{N}_{l_{0}}$ can be also guaranteed.

Indeed, this is so if the dimension of characteristic variety of the equation $\mathfrak{N}$ exceeds the dimension of characteristic variety of the pseudogroup $G$ (just by comparison of Poincaré polynomials for the corresponding symbolic modules). This latter condition is realized for infinite pseudogroups $G$ acting on jet-spaces $J^{\infty}(\pi)$ with "functional dimension" $\operatorname{dim}_{\mathbb{C}} \operatorname{Char}^{\mathbb{C}}(G)<\operatorname{rank}(\pi)$ and for (finitedimensional) Lie groups $G$ acting on equations $\mathfrak{N}$ of infinite type.

We will now argue that the theorem holds for general non-integrable (for both $G$ and $\mathfrak{N}$ ) case as well and show how the equation $\mathfrak{N}$ on submanifolds naturally appears. This is contained in the following three remarks.

1. When we consider the orbits of the pseudogroup $G$ even in the pure jetspace $J_{r}^{l}(M)$ (but maybe on equation) there are regular and singular orbits. The setup for constructing invariant differentiations requires to restrict to the former. In addition, the differential invariants as well as the Tresse derivatives are usually not defined on the whole space (for instance, because these absolute differential invariants can be obtained as ratio of two relative differential invariants). Thus we need to remove a closed nowhere dense subset of the jet-space. This subset 
(or its regular part) is a $G$-invariant equation $\mathfrak{N}$. And we need to apply the machinery to it. In turn, the orbits in it are divided into regular and singular, so that we get smaller equation etc.

2. If the equation $\mathfrak{N}$ or the pseudogroup $G$ are not integrable, then we must use the prolongation-projection scheme. Each time we obtain a set of compatibility conditions we project the equation $G_{k}\left(\right.$ or $\mathfrak{N}_{k}$ ) to obtain new equations of smaller order, which we prolong etc. When this concerns the pseudogroup, the space of differential invariants grows: To the existent invariants we add new. In addition, we can preserve existing invariant differentiations if we already possess some, adding only new generators - differential invariants $g_{m}, \ldots, g_{m+s}$.

Note that a shrink of $G$ results in a shrink of the characteristic variety $\operatorname{Char}^{\mathbb{C}}(G)$ and a shrink of $\mathfrak{N}$ results in a shrink of its prolongation. Therefore both regularity sets $\operatorname{Reg}_{l}^{1}$ and $\operatorname{Reg}_{l}^{2}$ can change and we should care that the density property for regular points is not lost. However by Cartan-Kuranishi theorem there is only a finite number of such shrinks in the process of prolongationprojection. On each of this step we add a finite number of differential invariants, which remain invariants during the rest of the process.

3. The Tresse derivatives as introduced in $\$ 1.3$ are invariant differentiations provided that $G$ and $\mathfrak{N}$ are integrable (this is not obvious from (7), but follows from the preceding formulas). But since we arrive to integrable equations in a finite number of steps, we will eventually get the required differentiations or observe finiteness of invariants. Thus even in non-integrable (but sufficiently regular) case Lie theorem holds.

\section{Invariants of geometric structures}

In this section we check the transitivity condition for the automorphisms pseudogroups of some basic geometric structures. Irreducible Lie pseudogroups were classified by E. Cartan ([1]). We consider at first these integrable pseudogroups. Examples of this section were mostly considered in our preceding paper [KL2], so the proofs will be omitted, though we indicate how to obtain the results from our cohomological machinery.

Note that the $l$-pseudogroup $G^{l}$ consists of the jets of diffeomorphisms preserving the structure to order $l$. So if the structure is non-integrable, then the prolongation-projection method changes the equation and the sub-pseudogroup $G^{j}$ can be different as embedded into $G^{k}$ and $G^{l}, j<\min (k, l)$ (so one should be careful with notations). We consider examples of the transformation pseudogroups of non-integrable structures at the end of the section.

In this section we suppose $\mathfrak{N}=J_{r}^{l}(M)$ unless the contrary is stated (thus $\left.h_{a}^{l}=S^{l} \mathfrak{t}_{a}^{*} \otimes \mathfrak{v}_{a}\right)$.

\subsection{General and volume preserving pseudogroups}

The general pseudogroup $G=\operatorname{Diff}_{\text {loc }}(M)$ is involutive. We have: $G^{l}=$ $D^{l}(M)$ for all $l$. In this case $\mathfrak{h}^{l}=($ Ann $\mathfrak{t}){ }_{\text {sym }} S^{l-1} T^{*} M \otimes T M+S^{l} T^{*} M \otimes \mathfrak{t}$. 
Complex (9) contains the sub-complex

$$
0 \rightarrow S^{l} T_{a}^{*} M \otimes \mathfrak{t}_{a} \rightarrow S^{l-1} T_{a}^{*} M \otimes \mathfrak{t}_{a} \otimes \mathfrak{t}_{a}^{*} \rightarrow S^{l-2} T_{a}^{*} M \otimes \mathfrak{t}_{a} \otimes \Lambda^{2} \mathfrak{t}^{*} \rightarrow \ldots
$$

and the quotient complex is exact. Indeed, it is the sum by $k$ of the complexes, each of which is $S^{l-k}(\mathrm{Ann} \mathfrak{t})$ tensorially multiplied by the exact complex

$0 \rightarrow S^{k} \mathfrak{t}_{a}^{*} \otimes T_{a} M / \mathfrak{t}_{a} \rightarrow S^{k-1} \mathfrak{t}_{a}^{*} \otimes T_{a} M / \mathfrak{t}_{a} \otimes \mathfrak{t}_{a}^{*} \rightarrow S^{k-2} \mathfrak{t}_{a}^{*} \otimes T_{a} M / \mathfrak{t}_{a} \otimes \Lambda^{2} \mathfrak{t}^{*} \rightarrow \ldots$

Since complex (12) has nontrivial only zero-cohomology group, which is isomorphic to $S^{l}\left(\right.$ Ann $\left.\mathfrak{t}_{a}\right) \otimes \mathfrak{t}_{a}$, the same holds for complex (9). Therefore we get $\mathfrak{O}^{l}=0$ for every $l$, whence all submanifolds $N$ are transversal.

Similarly if $\Omega$ is a volume form on $M$, the volume preserving pseudogroup $G=\operatorname{Diff}_{\text {loc }}(M, \Omega)$ is involutive: The only non-zero $\delta$-cohomology groups are $H^{0, j}(\mathfrak{g})$. Indeed, $G^{1}\left(a_{1}\right)=S L\left(T_{a} M\right)$, so $\mathfrak{g}_{1}=\operatorname{sl}\left(T_{a} M\right)$ and $\mathfrak{g}_{l}=S^{l} T_{a}^{*} M \otimes T_{a} M$ for $l>1$ as for the general pseudogroup. The homological calculations are similar and we again get $\mathfrak{D}^{l}=0$ for every $l$.

Thus for both pseudogroups all submanifolds of codimension $r$ are locally $G$-equivalent (the pseudogroups act transitively).

\subsection{Complex pseudogroup}

Let $G=\operatorname{Diffloc}_{\text {loc }}^{\mathbb{C}}(M)$ be a pseudogroup of local holomorphic transformations of a complex manifold $(M, J)$ of $\operatorname{dim}_{\mathbb{C}} M=n$. This group is also involutive, $\mathfrak{g}_{a}^{l}=S_{\mathbb{C}}^{l} T_{a}^{*} M \otimes_{\mathbb{C}} T_{a} M$. Condition (10) reads:

$$
\left(\begin{array}{c}
l+n-1 \\
l
\end{array}\right) \cdot 2 n \geq\left(\begin{array}{c}
l+2 n-r-1 \\
l
\end{array}\right) \cdot r .
$$

This holds true when $r \geq n$, but for $r<n$ it is wrong when $l>1$. Note though that the for $l=1$ the above inequality holds for all $0<r<n$. In this case (10) is not sufficient for 1-transversality of all $N$, but it is sufficient for submanifolds $N$ of general type at the point $a$. For $r \geq n$ we will show that a generic submanifold $N$ is transversal.

Suppose that $\mathfrak{t}_{a}$ contains no nontrivial $J$-invariant subspaces.

Let $T_{a} M=\mathfrak{t}_{a} \oplus J \mathfrak{t}_{a} \oplus \Pi_{a}$ be a (non-canonical) decomposition, where $\Pi$ is some $J$-invariant complement to the first two summands. We get the decomposition $\mathfrak{h}^{l}=(J \mathfrak{t})^{*} \circ S_{\mathbb{C}}^{l-1} T^{*} M \otimes_{\mathbb{C}} T M+\Pi^{*} \circ_{\mathbb{C}} S_{\mathbb{C}}^{l-1} T^{*} M \otimes_{\mathbb{C}} T M+S_{\mathbb{C}}^{l} T^{*} M \otimes \mathfrak{t}$. To be more precise we have two exact three-sequences ( $\sigma^{l}$ is given by the diagram):

$$
\begin{aligned}
0 & \rightarrow S_{\mathbb{C}}^{l} T^{*} M \otimes \mathfrak{t} \rightarrow \mathfrak{h}^{l} \rightarrow \sigma^{l} \rightarrow 0 \\
\| & \| \\
0 \rightarrow(J \mathfrak{t})^{*} \circ S_{\mathbb{C}}^{l-1} T^{*} M \otimes T M / \mathfrak{t} & \rightarrow \sigma^{l} \rightarrow \Pi^{*}{ }_{\mathbb{C}} S_{\mathbb{C}}^{l-1} T^{*} M \otimes_{\mathbb{C}} T M \rightarrow 0 .
\end{aligned}
$$

The Spencer sequences

$$
\ldots \stackrel{\delta}{\longrightarrow} g_{i+1} \otimes \Lambda^{j} \mathfrak{t}^{*} \stackrel{\delta}{\longrightarrow} g_{i} \otimes \Lambda^{j+1} \mathfrak{t}^{*} \stackrel{\delta}{\longrightarrow} \ldots
$$

with $g_{k}$ being equal $S_{\mathbb{C}}^{k} T^{*} M \otimes \mathfrak{t},(J \mathfrak{t})^{*} \circ S_{\mathbb{C}}^{k-1} T^{*} M \otimes T M / \mathfrak{t}$ or $\Pi^{*} o_{\mathbb{C}} S_{\mathbb{C}}^{k-1} T^{*} M \otimes \mathbb{C}$ $T M$ has vanishing positive cohomology groups $H^{i, j+1}=0, j \geq 0$. Hence the positive cohomology of (9) vanish too.

Thus Theorem[12 implies the transversality of $N$ with respect to $G$ : 
Proposition 17. The pseudogroup $G$ is l-transversal near $a_{l}=[N]_{a}^{l}$ iff:

1. $\mathfrak{t}_{a} \cap J \mathfrak{t}_{a}=\{0\}$ or $\mathfrak{t}_{a}+J \mathfrak{t}_{a}=T_{a} M$, when $l=1$,

2. $\mathfrak{t}_{a} \cap J \mathfrak{t}_{a}=\{0\}$, when $l>1$.

In particular, the complex pseudogroup $G$ acts transitively on local submanifolds $N \ni a$ of dimension $\operatorname{dim}_{\mathbb{R}} N \leq n$ with a generic 1 -jet at $a$.

On the other hand, a submanifold of dimension $\operatorname{dim}_{\mathbb{R}} N>n$ is never transversal. Namely, the intersection $\Pi_{a}=\mathfrak{t}_{a} \cap J \mathfrak{t}_{a} \neq\{0\}$ and so $N$ possesses an intrinsic geometry. Investigation of manifolds $N$ equipped with a complex structure on a distribution $\Pi$ is the subject of Cauchy-Riemann geometry.

The space of covariants $\mathfrak{D}_{a}^{l}$ is now non-zero. Thus there are differential invariants of the action. They are the curvatures of Cartan-Chern-Moser [CM]. Fixing the curvatures we get a smaller class $\mathfrak{N}$ of submanifolds, on which the action will be eventually transitive.

Another important class $\mathfrak{N}$ consists of all complex submanifolds $N \subset M$ of $\mathbb{C}$-codimension $r$. This class is $l$-transversal for every $l$, and so is transitive.

\subsection{Symplectic pseudogroup}

Consider a symplectic manifold $(M, \omega)$ of dimension $2 n$. Let $G=\operatorname{Symp}(M, \omega)$ be its (pseudo)group of symplectomorphisms. This pseudogroup is involutive, i.e. $H^{i, j}(\mathfrak{g})=0$ for $i>0$.

Using the identification $T M \stackrel{\omega}{\simeq} T^{*} M$ we write the symbols $\mathfrak{g}_{a}^{l}=S^{l+1} T_{a}^{*} M \subset$ $S^{l} T_{a}^{*} M \otimes T_{a}^{*} M$, understood as homogeneous generating functions (Hamiltonians) of degree $l+1$.

Condition (10) for $l$-transversality in this case always holds:

$$
\left(\begin{array}{c}
l+2 n \\
l+1
\end{array}\right) \geq\left(\begin{array}{c}
l+2 n-r-1 \\
l
\end{array}\right) \cdot r,
$$

Thus we get no restrictions on the dimension of a submanifold $N$.

We consider the case of even-dimensional $\mathfrak{t}, \operatorname{dim} \mathfrak{t}=2 r$ (the odd-dimensional case is similar). Suppose that $\omega^{r}$ is non-degenerate on $\mathfrak{t}$.

Let $T M=\mathfrak{t} \oplus \Pi$ be the direct $\omega$-orthogonal decomposition, $\Pi=\mathfrak{t}^{\perp \omega}$. Under the identification $T M \simeq T^{*} M$ we have: $\mathfrak{t} \simeq \mathfrak{t}^{*}, \Pi \simeq \Pi^{*}$. So we calculate $\mathfrak{h}^{1}=$ $S^{2} \mathfrak{t}^{*}+S^{2} \Pi^{*}$. For the prolongations we have $\mathfrak{h}^{l}=S^{l+1} \mathfrak{t}^{*}+S^{2} \Pi^{*}$ osym $_{\text {sy }} S^{l-1} T^{*} M$.

Therefore complex (9) is the sum of the exact sequence

$$
0 \rightarrow S^{l+1} \mathfrak{t}^{*} \rightarrow S^{l} \mathfrak{t}^{*} \otimes \mathfrak{t}^{*} \rightarrow S^{l-1} \mathfrak{t}^{*} \otimes \Lambda^{2} \mathfrak{t}^{*} \rightarrow \ldots
$$

and the tensor product of $S^{2} \Pi^{*}$ with the complex

$$
0 \rightarrow S^{l-1} T^{*} M \rightarrow S^{l-2} T^{*} M \otimes \mathfrak{t}^{*} \rightarrow S^{l-3} T^{*} M \otimes \Lambda^{2} \mathfrak{t}^{*} \rightarrow \ldots,
$$

which (cf. (12) ) has only one nontrivial cohomology group $H^{l-1,0} \simeq S^{l-1} \Pi^{*}$.

Thus we again obtain a transitivity result: 
Proposition 18. $G$ acts l-transversally for all $l \geq 0$ (and hence transitively) near $a_{l} \in J_{r}^{l}(M)$ iff the restriction of $\omega$ to $\mathfrak{t}_{a}=a_{1}$ is of maximal rank.

The obtained fact is equivalent to a particular case of Weinstein-Givental theorem ( $\mathrm{AG}]$ ). To obtain the more general case we should allow various ranks for the restrictions $\left.\omega\right|_{N}$. Then the transversality fails and we get a 1-variant, which is obviously the rank (or dimension of $\operatorname{Ker}\left(\left.\omega\right|_{N}\right)$ ). Fixing it we obtain the transversality for the corresponding equation $\mathfrak{N}$ on submanifolds.

Finally consider the class $\mathfrak{N}$ of isotropic or co-isotropic submanifolds. Similar calculations show that $G$ acts on it $l$-transversally for every $l$.

\subsection{Contact pseudogroup}

Consider a contact manifold $\left(M, \Pi^{2 n}\right), \operatorname{dim} M=2 n+1$, and denote by $\nu=T M / \Pi$ the normal. Let $G=\operatorname{Cont}(M, \Pi)$ be the (pseudo) group of contact transformations. Again the pseudogroup is involutive. Its Lie algebra consists of contact vector fields $X_{f}$, which are determined by generating functions (Hamiltonians) $f \in C^{\infty}(M) \otimes \nu$.

Necessary condition (10) for $l$-transversality again holds always:

$$
\left(\begin{array}{c}
l+2 n+1 \\
l+1
\end{array}\right) \geq\left(\begin{array}{c}
l+2 n-r \\
l
\end{array}\right) \cdot r,
$$

Thus no restriction on the dimension of a submanifold $N$ is imposed.

A choice of a non-zero section of $\nu$ is equivalent to a choice of a contact form $\alpha \in C^{\infty}(\operatorname{Ann}(\Pi) \backslash 0), \alpha \wedge d \alpha^{n} \neq 0$. Then the Hamiltonian is scalar-valued, $f \in C^{\infty}(M)$, and the contact field is uniquely given by

$$
\alpha\left(X_{f}\right)=f, d \alpha\left(\cdot, X_{f}\right)=\left.d f\right|_{\Pi} .
$$

In Darboux coordinates $(q, u, p), \alpha=d u-p_{i} d q^{i}$, we have:

$$
X_{f}=\mathcal{D}_{q^{i}}(f) \partial_{p_{i}}-\partial_{p_{i}}(f) \mathcal{D}_{q^{i}}+f \partial_{u}, \quad \text { where } \mathcal{D}_{q^{i}}=\partial_{q^{i}}+p_{i} \partial_{u} .
$$

Note that fixing $\alpha$ is equivalent to the splitting $T_{a} M=\Pi_{a} \oplus \nu_{a}$, where the first summand is symplectic and the second is Euclidean 1-dimensional. To describe the symbol $\mathfrak{g}_{a}^{l}$, we identify $T M \simeq T^{*} M$ summand-wise via the symplectic structure on $\Pi$ and the Euclidean structure on $\mathfrak{v}$. Then we get:

$$
\mathfrak{g}^{l} \simeq S^{l} \nu^{*} \oplus \sum_{i>0} S^{i} \Pi^{*} \otimes S^{l+1-i} \nu^{*} \simeq S^{l+1} T^{*} M .
$$

In fact, order $l$ contact fields $X_{f}$ are determined by Hamiltonians $f$ of order $(l+1)$ in all variables except the pure power of $u$, where the degree is $l$.

Thus the cohomological calculations are quite similar to the symplectic case and we get:

Proposition 19. The action of $G$ is l-transversal near $a_{l} \in J_{r}^{l}(M)$ for all $l \geq 0$ iff $\mathfrak{t}_{a}=T_{a} N$ is transversal to the contact plane $\Pi_{a}$ and the induced structure on $\Pi_{a}^{N}=\Pi_{a} \cap T_{a} N$ from the canonical conformally-symplectic structure on $\Pi$ is maximally nondegenerate. This means that 
- if $\operatorname{dim} \Pi_{a}^{N}=2 r$, then $\left.(d \alpha)^{r}\right|_{\Pi_{a}^{N}} \neq 0$.

- if $\operatorname{dim} \Pi_{a}^{N}=2 r+1$, then $\operatorname{rank}\left(\left.d \alpha\right|_{\Pi_{a}^{N}}\right)=2 r$.

These conditions are equivalent to the claim that through every point close to $a \in N$ there passes an isotropic submanifold of dimension no greater than $r$.

As in the symplectic case we note that $\operatorname{rank}\left(\left.(d \alpha)^{r}\right|_{\Pi^{N}}\right)$ is a 1-variant, fixing which we get transversality. This is a particular case of the contact WeinsteinGivental theorem: If two local submanifolds of codimension $r$ of a contact manifold $(M, \Pi)$ have isomorphic restrictions of the contact structure $\left(N, \Pi^{N}\right)$, then they have contactomorphic neighborhoods.

At last, as in $\S 3.3$ a particular case says that restricting to the class $\mathfrak{N}$ of isotropic submanifolds of fixed dimension, we get transversality of the $G$-action.

\subsection{Riemannian pseudogroup}

Consider at first the isometry pseudogroup of the Euclidean space $\mathbb{R}^{n}$. It integrates to the group $G=O(n) 入 \mathbb{R}^{n}$. The pseudogroup is of finite type and $\mathfrak{g}_{a}^{l}=0$ for $l \geq 2\left([\mathrm{Ko})\right.$. This means that we have plenty of covariants $\mathfrak{O}_{a}^{l}=h_{a}^{l}$, $l \geq 2$. Thus the transversality is absent for $l \geq 2$, but the action is 1 -transversal near each 1 -jet $\varphi_{1}$.

Consider now a Riemannian manifold $\left(M^{n}, q\right)$ and let $G$ be the isometry pseudogroup. If the sectional curvature is constant, everything is the same is above. But in general case the pseudogroup $G$ is no longer integrable.

For $l=1,2$ the group $G^{l}$ is the same as in Euclidean case. But not every point $\varphi_{2}$ has a prolongation to $G^{3}$. Indeed, $G_{a}^{1, \text { new }}=\rho_{3,1}\left(G_{a}^{3}\right) \subset G_{a}^{1}$ consists of linear isometries from the orthogonal group $O\left(T_{a} M, q\right)$ preserving the Riemannian curvature tensor $R_{q}$.

Moreover, the prolongation-projection method reduces soon the pseudogroup to the unit element: For a generic Riemannian structure $q$ the pseudogroups $G^{l}$ consists of the identity only, when $l>3$ or $l=3, n>2$.

Proposition 20. The action of $G$ is not transversal near any $l$-jet for $l>1$.

In fact, various intrinsic and extrinsic curvatures are $l$-variants. More generally, all differential invariants can be obtained from the curvatures via LeviCivita connection operator. We will obtain transitivity of the action on the equation, which gives constancy of all these invariants. More generally they give a solution to the equivalence problem.

\subsection{Almost complex pseudogroup}

Consider now an almost complex manifold $\left(M^{2 n}, J\right), J^{2}=-\mathbf{1}$. The pseudogroup $G^{l}$ consists of all $J$-holomorphic $l$-jets: $J \circ \varphi_{l}=\varphi_{l} \circ J$. It is nonintegrable whenever the almost complex structure $J$ is non-integrable. Let us investigate this case. 
Denote by $N_{J} \in \operatorname{Hom}_{\overline{\mathbb{C}}}\left(\Lambda^{2} T M, T M\right)$ the Nijenhuis tensor of the structure $J$. This is the obstruction for $J$ to be integrable (the notation means that it is (2,1)-tensor $J$-antilinear by each argument).

For $l=1$ we have: $G_{a}^{1}=\mathrm{GL}_{\mathbb{C}}\left(T_{a} M\right)$, as in the complex case. The prolongation $G^{2}$ does not exist over all points of $G^{1}$. Using the prolongation-projection method we obtain:

$G_{a}^{1, \text { new }}=\rho_{2,1}\left(G_{a}^{2}\right)=\left\{\Phi \in T_{a}^{*} M \otimes T_{a} M \mid J \circ \Phi=\Phi \circ J, N_{J} \circ(\Phi \wedge \Phi)=\Phi \circ N_{J}\right\}$.

A symmetric torsion-free connection $\nabla$ on $M$ gives a decomposition of the 2jet $\varphi_{2} \in G^{2}$ into components $\left(a, \Phi, \Phi^{(2)}\right)$. The last terms $\Phi^{(2)} \in \mathfrak{F}\left(\varphi_{1}\right)$ for $\varphi_{1}=(a, \Phi)$ are jointly described by the formula:

$\left\{\Phi^{(2)} \in S^{2} T_{a}^{*} M \otimes T_{a} M, J \Phi^{(2)}(\xi, \eta)-\Phi^{(2)}(J \xi, \eta)=\Phi \circ \nabla_{\eta}(J)(\xi)-\nabla_{\Phi \eta}(J)(\Phi \xi)\right\}$,

Thus $\mathfrak{g}_{a}^{2}=S^{2} T_{a}^{*} M \otimes_{\mathbb{C}} T_{a} M$ as in the complex case, but for a smaller set of $\varphi_{1}$. The 2-pseudogroup $G^{2}$ is not 2-integrable in general. Proof of these facts, as well as a description of the projection $\rho_{l, l-1}: G_{a}^{l} \rightarrow G_{a}^{l-1}$ are contained in Kr1.

It can be shown, see $\underline{\mathrm{Kr} 2}$, that for a generic structure $J$ the set $G^{2}$ consists of identity for $n>3, G^{3}$ consists of identity for $n>2$ and $G^{4}$ is the identity (we ignore the case $n=1$ corresponding to always integrable $J$ ). The analysis of pseudoholomorphic invariants for jets of submanifolds based on the classification of Nijenhuis tensors ( $\mathrm{Kr} 2$ ) results in:

Proposition 21. Let $(M, J)$ be an almost complex manifold with a generic nonintegrable structure $J$ and $n>1$. For $l=1$ the transversality is described by condition 1 of proposition [17 For $l=2$ no 2-jet is transversal save for the case $n=2$ and $\operatorname{dim}_{\mathbb{R}} N=1$. The transversality is absent for $l=3$ and higher.

So in the case of generic almost complex pseudogroup we have: $\mathfrak{g}^{l}=0$ for big $l$, whence plenty of covariants $\mathfrak{O}_{a}^{l}$ and no transversality. All the differential invariants here can be obtained from the Nijenhuis tensor $N_{J}$ Kr1].

\section{Equivalence of differential equations}

Let $\pi$ be a vector bundle. A submanifold in $J^{k} \pi$ can be identified with a differential equation (actually a system of equations, but we will just say "equation"). For regularity purposes we assume it is a subbundle w.r.t. all $\pi_{j, j-1^{-}}$ projections, i.e. we have a sequence $\mathcal{E}_{j} \subset J^{j} \pi$ of submanifolds and projections $\pi_{j, j-1}: J^{j} \pi \rightarrow J^{j-1} \pi, j \leq k$, forming vector bundles.

Consider two such differential equations $\mathcal{E} \subset J^{k} \pi$ and $\mathcal{E}^{\prime} \subset J^{k} \pi^{\prime}$ and two points $x_{k}, x_{k}^{\prime}$. There exists a Lie transformation $\varphi: J^{\epsilon} \pi \rightarrow J^{\epsilon} \pi^{\prime}$, for which

$\varphi^{(k)}\left(x_{k}\right)=x_{k}^{\prime}$. So we reduce the problem to the case, when both equations $\mathcal{E}$ and $\mathcal{E}_{\varphi}^{\prime}=\left(\varphi^{-1}\right)^{(k)}\left(\mathcal{E}^{\prime}\right)$ live in one space $J^{k} \pi$. Then we try to identify $\mathcal{E}$ to $\mathcal{E}_{\varphi}^{\prime}$ by means of a Lie transformation.

In this section we call Lie pseudogroup $G$ the pseudogroup of Lie transformations on the jet-spaces $J^{k} \pi$. Lie-Bäcklund theorem ([KLV]) states that such 
a transformation is lifted from a diffeomorphism of $J^{0} \pi$ in the case $\operatorname{rank} \pi>1$ (point transformations) or from a contact diffeomorphism of $J^{1} \pi$ for $\operatorname{rank} \pi=1$ (contact transformations). So Lie transformations are lifted from $J^{\epsilon} \pi$, where $\epsilon=\max (0,2-\operatorname{rank} \pi)$.

\subsection{Formally transitive actions of the Lie pseudogroup}

For the Lie pseudogroup $G$ of point transformations or contact transformations (depending on $\epsilon$ ) we provide calculation of the symbols in Appendix B This implies ( KL2 ) that the dimension of the symbols grow as:

$$
\operatorname{dim} \mathfrak{g}^{l} \sim n_{0} \cdot \frac{l^{n_{0}-1}}{\left(n_{0}-1\right) !}, \quad n_{0}=\operatorname{dim} J^{0} \pi
$$

in the case of point transformations $(\epsilon=0)$ and

$$
\operatorname{dim} \mathfrak{g}^{l} \sim \frac{l^{n_{1}-1}}{\left(n_{1}-1\right) !}, \quad n_{1}=\operatorname{dim} J^{1} \pi
$$

in the case of contact transformations $(\epsilon=1)$. Using the necessary condition (10) we obtain the following characterization of equations $\mathcal{E}$ on which the Lie pseudogroup $G$ acts transitively.

Theorem 22. The only transversal equations $\mathcal{E} \subset J^{k} \pi$ w.r.t. the Lie transformation pseudogroup are the following:

1. $u_{x}^{\prime}=\varphi(x, u), x \in \mathbb{R}, u \in \mathbb{R}^{n}$.

2. $u_{x^{i}}^{\prime}=\varphi_{i}(x, u), i=1, \ldots, n, x \in \mathbb{R}^{n}, u \in \mathbb{R}$.

3. $w_{z}^{\prime}=\varphi_{1}(z, \bar{z}, w, \bar{w}), w_{\bar{z}}^{\prime}=\varphi_{2}(z, \bar{z}, w, \bar{w}), z, w \in \mathbb{C}$.

4. $u_{x^{i}}^{\prime}=\varphi_{i}\left(x, u, u_{x^{s+1}}^{\prime}, \ldots, u_{x^{n}}^{\prime}\right), 1 \leq i \leq s<n, x \in \mathbb{R}^{n}, u \in \mathbb{R}$.

5. $u_{x x}^{\prime \prime}=\varphi\left(x, u, u_{x}^{\prime}\right), x \in \mathbb{R}, u \in \mathbb{R}$.

Let us comment the five cases of the theorem and indicate for which $\varphi$ we actually have the transversality. Let $k$ denote the order of the equation $\mathcal{E}, n$ the dimension of the base of $\pi$ and $r$ the rank of $\pi$ (dimension of the fiber).

1. $n=k=1$. A submanifold $\mathcal{E} \subset J^{1}(1, r)$ of codimension $r$ is a determined system of ODEs. Due to our regularity assumptions they are of main type, so can be written as in the theorem. By the existence and uniqueness theorem locally all such systems are equivalent, i.e. the pseudogroup acts transversally on them. In this case $\mathcal{E}$ is integrable.

2. $r=k=1$. Here $\mathcal{E} \subset J^{1}(n, 1)$ of codimension $n$ is diffeomorphically projected by $\pi_{0}$ to $J^{0} \pi$. Through every point $x_{0}=\pi_{0}\left(x_{1}\right)$ an $n$-plane $L\left(x_{1}\right)$ passes. Their collection is the image of the Cartan distribution $\mathcal{C}_{\mathcal{E}}$ on $\mathcal{E}$. The obtained rank $n$ distribution on the manifold $\mathcal{E}^{n+1}$ is generically non-integrable and is either contact or even-contact. In both cases we get transversality and 
local equivalence of all such equations. Thus in this case we take $\varphi_{i}$ such that $\mathcal{E}$ is maximally non-integrable.

3. $k=1, n=r=2$. Here the functions should again be taken generic. Then $\mathcal{E}$ is non-integrable and we obtain transitivity of the action.

Note that in all the above 3 cases $\pi_{1,0}: \mathcal{E} \rightarrow J^{0} \pi$ is a diffeomorphism, so that we have the distribution $d \pi_{1,0}\left(\mathcal{C}_{\mathcal{E}}\right)$ on $J^{0} \pi$. The described cases correspond to the known distributions $\Pi$ without moduli: (1) Line field of $\operatorname{rank}(\Pi)=$ 1 ; (2) Contact or even-contact distribution of $\operatorname{corank}(\Pi)=1$; (3) The Engel distribution of $\operatorname{rank}(\Pi)=\operatorname{corank}(\Pi)=2$ on a four-dimensional manifold.

4. $k=1$ and $\mathcal{E} \subset J^{1}(n, 1)$ is a submanifold in a contact manifold. As in $\$ 3.4$ we see that $\operatorname{PDE} \mathcal{E} \subset J^{1} \pi$ of $\operatorname{dim} \mathcal{E}=d$ is transversal w.r.t. the Lie pseudogroup at $x_{1} \in \mathcal{E}$ iff there are no integral manifolds of the contact structure $\Pi$ of dimension greater than $\left[\frac{d-1}{2}\right]$. Note that the induced distribution $\Pi \cap T \mathcal{E}$ on $\mathcal{E}$ has always integral submanifolds $L$ of dimension $\left[\frac{d-1}{2}\right]$. If $\pi_{1}: L \rightarrow L_{0}$ is a diffeomorphism, the submanifold has the form $j_{1} s\left(L_{0}\right)$ for some section $s$ of the bundle $\pi$. So transversality of $\mathcal{E}$ means there are no "partial solutions" $s: L_{0} \rightarrow J^{0} \pi, j_{1} s\left(L_{0}\right) \subset \mathcal{E}$, of dimension greater than the minimal possible.

5. $k>1, n=1$. If $\mathcal{E}_{1}=\pi_{k, 1}(\mathcal{E}) \subset J^{1} \pi$ is proper then either there does not exist the prolongation $\mathcal{E}_{1}^{(1)}$ or the equation $\mathcal{E}_{1}$ and hence $\mathcal{E}$ is not transversal. So we consider $\mathcal{E}_{1}=J^{1} \pi$ and then $\pi_{2,1}: \mathcal{E}_{2} \rightarrow J^{1} \pi$ is a diffeomorphism. In this case we have $l$-transversality for every $l$. Note that here $\mathcal{E}$ is integrable.

The last case corresponds to a known result of S. Lie: All the scalar ordinary differential equations of the second order are contact equivalent. This case is equivalent to a Legendrian foliation of the contact 3 -manifold $J^{1}(1,1)$.

In fact, locally all Legendrian foliations of a contact manifold $J^{1}(n, 1)$ are equivalent, but only for $n=1$ (corresponding to S. Lie's theorem) the corresponding equation $\mathcal{E}$ is generic. Otherwise, an additional assumption of integrability should be imposed on $\mathcal{E}$. For such class of equations we get the following result:

Proposition 23. The Lie transformations pseudogroup acts transitively on the class $\mathfrak{N}$ of integrable equations $\mathcal{E} \subset J^{1+\epsilon} \pi$, such that $\pi_{1+\epsilon, \epsilon}: \mathcal{E} \stackrel{\sim}{\rightarrow} J^{\epsilon} \pi$ is a diffeomorphism.

Indeed, for $\epsilon=1$ we have a Legendrian foliation of $J^{1} \pi$ and for $\epsilon=0$ just a foliation of $J^{0} \pi$.

\subsection{Formally intransitive actions of the Lie pseudogroup}

In all other cases except for the above five, the differential equations have invariants. Their growth is governed by the cohomology of covariants $H^{*, *}(\mathfrak{O})$.

By Lie-Tresse theorem the invariants of the differential equation have a finite set of generators. Let us consider some examples.

1. Consider a scalar second order ODE $y^{\prime \prime}=u\left(x, y, y^{\prime}\right)$. By the results of 4.1 the pseudogroup of contact transformations acts transitively. For point 
transformations there are differential invariants ( $\operatorname{Tr} 2$ ). The following functions are the basic differential invariants and the others are obtained by certain invariant differentiations $\mathrm{C} 2$.

$$
I_{1}=u_{1111}, \quad I_{2}=u_{x x 11}-u_{1} u_{x 11}-4 u_{x 01}+4 u_{1} u_{01}-3 u_{0} u_{11}+6 u_{00}
$$

Here we have denoted the differentiation by $y^{(i)}$ with subindex $i$, so that we have $u_{x 1}=\frac{\partial^{2} u}{\partial x \partial y^{\prime}}$ etc.

2. Consider the action of $\mathrm{SL}(3)$ on $\mathbb{R} P^{2}$ by projective transformations:

$$
\left[z_{0}: z_{1}: z_{2}\right] \mapsto\left[(A z)_{0}:(A z)_{1}:(A z)_{2}\right], \quad A \in \mathrm{SL}(3), z \in \mathbb{R}^{3} .
$$

This action lifts to higher jets $J_{1}^{k}\left(\mathbb{R} P^{2}\right)$. It is transitive near regular orbits for $k \leq 6$. For $k=6$ it becomes effective. The first differential invariants $I_{7}$ occurs for $k=7$. It equals $\left(\theta_{8}\right)^{3} /\left(\theta_{3}\right)^{8}$, where $\theta_{3}$ and $\theta_{8}$ are some basic relative differential invariants ( $\mathrm{La}$; the index $k$ in $\theta_{k}$ refers to the factor under transformations of this relative invariant). Thus we have an invariant differentiation $\hat{\partial}=\hat{\partial}_{I_{7}}$.

The next relative differential invariant is obtained via a bracket of these $\theta_{3}$ and $\theta_{8}(\underline{W})$ and using it we can obtain a new differential invariant $I_{8}$ of order 8. There will be exactly one differential invariant of order $k \geq 9$ and each of them is obtained by the iterated Tresse derivative: $I_{k}=\hat{\partial}^{k-8}\left(\bar{I}_{8}\right)$.

3. Monge-Ampère equations with two variables

$$
\alpha_{0}+\alpha_{1} u_{x x}+\alpha_{2} u_{x y}+\alpha_{3} u_{y y}+\alpha_{4} \cdot\left(u_{x x} u_{y y}-u_{x y}^{2}\right)=0,
$$

$\alpha_{i}=\alpha_{i}\left(x, y, u, u_{x}, u_{y}\right)$, can be represented geometrically as effective 2 -forms on the contact manifold $J^{1}\left(\mathbb{R}^{2}\right)$. This gives a possibility to construct an invariant frame on the equation ( $\{e\}$-structure) and so to describe all differential invariants ( Kr3, KLR $)$.

4. If we consider classification of non-integrable equations, then their Weyl tensors are differential invariants w.r.t. Lie pseudogroup of transformations of the jets-space, [KL1. Curvatures for geometric structures are particular cases. Note that prolongation-projection method produces an integrable equation from a given non-integrable, but the invariants obtained from the Weyl tensors remain differential invariants for the new equation.

Let us mention also another related classification problem. Given a differential equation $\mathcal{E}$ we can consider its pseudogroup of symmetries, i.e. such transformations from $G$ that map $\mathcal{E}$ to itself.

These Lie transformations are extrinsic symmetries for the differential equations. If an equation is not normal ([KLV]), there may exist also intrinsic symmetries, which cannot be obtained from the extrinsic ones. Considering a symmetry pseudogroup $G \subset \operatorname{Sym}(\mathcal{E})$ of the $\operatorname{PDE} \mathcal{E}$, we ask about equivalence problem for the solutions. The differential invariants for this problem are important for the integrability of the given PDE.

For generic $\mathcal{E}$ the symmetry pseudogroup is trivial (this is possible to show as we did in 3.6 with geometric structures). But for many important equations $\operatorname{Sym}(\mathcal{E})$ is sufficiently big $([\mathrm{KLV}])$ and the problem is interesting and non-trivial. 


\section{A. Basics from the geometric theory of PDEs}

Here we briefly recall some fundamental geometric notions of the jet-spaces (see [GS1, KLV] Gu, Ly, KL1] for details).

\section{A.1. Prolongations and projections}

A PDEs system of pure order $k$ is usually represented as a smooth subbundle $\left.\mathcal{E} \subset J^{k} \pi(\underline{\mathrm{KLV}}]\right)$. This means that non-regular points are removed and all equations in the system have pure order $k$. We extend this for different orders.

By a differential equation (system) of maximal order $k$ we mean a sequence $\mathcal{E}=\left\{\mathcal{E}_{l}\right\}_{-1 \leq l \leq k}$ of submanifolds $\mathcal{E}_{l} \subset J^{l} \pi$ with $\mathcal{E}_{-1}=B$ (base of $\pi$ ), $\mathcal{E}_{0}=$ $J^{0} \pi=E_{\pi}$ such that for all $0<l \leq k$ the following conditions hold:

1. $\pi_{l, l-1}^{\mathcal{E}}: \mathcal{E}_{l} \rightarrow \mathcal{E}_{l-1}$ are smooth fiber bundles.

2. The first prolongations $\mathcal{E}_{l-1}^{(1)}$ are smooth subbundles of $\pi_{l}$ and $\mathcal{E}_{l} \subset \mathcal{E}_{l-1}^{(1)}$.

We remark that in the jets of sections (contrary to the jets of submanifolds) we have also the projections to the base $\pi_{k}: J^{k} \pi \rightarrow B$.

Denote by $\tau_{x}$ the tangent space to the base $B$ of $\pi$ at the point $x=\pi_{k}\left(x_{k}\right)$ and by $\nu_{x_{0}}$ the tangent to the fiber at $x_{0}=\pi_{k, 0}\left(x_{k}\right)$. Let also $F\left(x_{k}\right)$ be the $\pi_{k+1, k}$-fiber and $v_{x_{1}}=T_{x_{1}}\left(F\left(x_{0}\right)\right)$.

Consider a point $x_{k} \in \mathcal{E}_{k}$ with $x_{l}=\pi_{k, l}\left(x_{k}\right)$ for $l<k$ and $x=x_{-1}$. It determines a symbolic system $g \subset S \tau_{x}^{*} \otimes \nu_{x}$ by the formula

$$
g_{l}=T_{x_{l}}\left[\left(\pi_{l, l-1}^{\mathcal{E}}\right)^{-1}\left(x_{l-1}\right)\right] \subset S^{l} \tau_{x}^{*} \otimes \nu_{x}
$$

for $l \leq k$ and $g_{l}=g_{k}^{(l-k)}$ for $l>k$. The conditions above imply that the symbols $g_{l}$ form smooth vector bundles over $\mathcal{E}_{l}$ and that $g_{l} \subset g_{l-1}^{(1)}$ for $l \leq k$. We call such collection of subspaces $\left\{g_{k}\right\}$ symbolic systems.

The Spencer $\delta$-complex for PDEs system $\mathcal{E}$ at a point $x_{k} \in \mathcal{E}_{k}$ is the Spencer complex for its symbolic system at this point:

$$
\cdots \rightarrow g_{i+1} \otimes \Lambda^{j-1} \tau_{x}^{*} \stackrel{\delta}{\longrightarrow} g_{i} \otimes \Lambda^{j} \tau_{x}^{*} \stackrel{\delta}{\longrightarrow} g_{i-1} \otimes \Lambda^{j+1} \tau_{x}^{*} \stackrel{\delta}{\rightarrow} \cdots
$$

The corresponding $\delta$-cohomology is denoted by $H^{i, j}\left(\mathcal{E} ; x_{k}\right)$.

We define regular PDEs system of maximal order $k$ as a submanifold $\mathcal{E}=$ $\mathcal{E}_{k} \subset J^{k} \pi$ "cofiltered" by $\mathcal{E}_{l}$ (property 1 above) and such that the symbolic system and the Spencer cohomology form graded bundles over it.

Define the Cartan distribution on the space $J^{k} \pi$ by the formula: $\mathcal{C}_{k}\left(x_{k}\right)=$ $\left(d \pi_{k, k-1}\right)^{-1} L\left(x_{k}\right)$. It induces the Cartan distribution on $\mathcal{E}_{k}: \mathcal{C}_{\mathcal{E}_{k}}=\mathcal{C}_{k} \cap T \mathcal{E}_{k}$.

A system of different orders should be investigated for formal integrability successively by the maximal order $k$. If some prolongation $\mathcal{E}_{k}^{(1)}$ is not regular, its projections $\left\{\pi_{k+1, l}\left(\mathcal{E}_{k+1}\right)\right\}_{l \leq k}$, form a new system of maximal order $k$. Taking the regular part one continues with prolongations. The process stops in a finite number of steps by Cartan-Kuranishi theorem on prolongations: There exists a number $k_{0}$ such that $\mathcal{E}_{k}^{(1)}=\mathcal{E}_{k+1}$ for all $k \geq k_{0}$. 


\section{A.2. Characteristics}

Consider the dual to $g$ system $g^{*}=\oplus g_{k}^{*}$. If $g$ is a symbolic system, then $g^{*}$ is an $S \tau$-module (as before $S \tau=\oplus S^{i} \tau$ and $\tau=\tau_{x}$ with "frozen" $x \in B$ ) with the structure given by

$$
(v \cdot \kappa) p=\kappa\left(\delta_{v} p\right), v \in S \tau, \kappa \in g^{*}, p \in g .
$$

This module, called the symbolic module, is Noetherian and the Spencer cohomology of $g$ dualizes to the Koszul homology of $g^{*}$.

The characteristic ideal is defined by $I(g)=\operatorname{ann}\left(g^{*}\right) \subset S \tau$. The affine characteristic variety of $g$ (or of $\mathcal{E}$ ) is the set of $v \in \tau^{*} \backslash\{0\}$ such that for every $k$ there exists a $w \in N \backslash\{0\}$ with $v^{k} \otimes w \in g_{k}$. This is a conical affine variety. If we consider its complexification and then projectivization, then we get the characteristic variety $\operatorname{Char}^{\mathbb{C}}(g) \subset P^{\mathbb{C}} \tau^{*}$.

Relation of characteristic variety to the characteristic ideal is given by the formula:

$$
\operatorname{Char}^{\mathbb{C}}(g)=\left\{p \in P^{\mathbb{C}} \tau^{*} \mid f\left(p^{k}\right)=0 \forall f \in I_{k}, \forall k\right\} .
$$

Note that the dimension of affine characteristic variety equals the Chevalley dimension of the symbolic module. Recall also that a sequence of elements $f_{1}, \ldots, f_{s} \in S \tau$ is called regular if $f_{i}$ is not a zero divisor in the $S \tau$-module $g^{*} /\left(f_{1}, \ldots, f_{i-1}\right) g^{*}$.

\section{A.3. Horizontal differential and generalizations}

The horizontal differential $\hat{d}: C^{\infty}\left(J^{k} \pi\right) \rightarrow \Omega^{1}\left(J^{k+1} \pi\right)$ is defined by the properties:

$$
\text { 1. }\left.\hat{d} f\right|_{\pi_{k+1}^{-1}(x)}=0, \quad \text { 2. }\left.\hat{d} f\right|_{j_{k+1}(s)}\left(x_{k+1}\right)=\left.d f\right|_{j_{k}(s)}\left(x_{k}\right)
$$

for any section $s$ of $\pi$ with $j_{k+1}(s)(x)=x_{k+1}, \pi_{k+1, k}\left(x_{k+1}\right)=x_{k}$. In local coordinates we can write:

$$
\hat{d} f=\sum \mathcal{D}_{i}(f) d x^{i}
$$

This can be used as a definition of the total derivative operators $\mathcal{D}_{i}$.

Indeed, choosing local coordinates $x^{i}$ (note the placement of indices) on the base of $\pi$ and $u^{j}$ on fibers, we obtain canonically the coordinates $\left(x^{i}, p_{\sigma}^{j}\right)_{0 \leq|\sigma| \leq k}$ on $J^{k} \pi$, where $p_{\sigma}^{j}\left([u]_{x}^{k}\right)=\frac{\partial^{|\sigma|} u^{j}}{\partial x^{\sigma}}$. Then the operator of total derivative $\mathcal{D}_{i}$ : $C^{\infty}\left(J^{k} \pi\right) \rightarrow C^{\infty}\left(J^{k+1} \pi\right)$ has the form:

$$
\mathcal{D}_{i}=\partial_{x^{i}}+\sum_{j ; \sigma} p_{\sigma+1_{i}}^{j} \partial_{p_{\sigma}^{j}}
$$

For a multiindex $\sigma=\left(i_{1}, \ldots, i_{n}\right)$ we define $\mathcal{D}_{\sigma}=\mathcal{D}_{1}^{i_{1}} \cdots \mathcal{D}_{n}^{i_{n}}$. If $l=|\sigma|$ is the length of the multi-index $\sigma$, then $\mathcal{D}_{\sigma}: C^{\infty}\left(J^{k} \pi\right) \rightarrow C^{\infty}\left(J^{k+l} \pi\right)$. 
If we consider the jet-space $J_{r}^{k}(\mathfrak{M}), \mathfrak{M}=J^{0} \pi=E_{\pi}, r=\operatorname{rank}(\pi)$, then $J^{k} \pi \hookrightarrow J_{r}^{k}(\mathfrak{M})$ is an open dense subset. In fact, choosing local coordinates $(x, u)$ on $\mathfrak{M}$ we identify it locally with $\pi$ and so the described embedding can be considered as a local chart. Even though the notions of total derivative and horizontal differential are not defined on $J_{r}^{k}(\mathfrak{M})$, we explained in 1.3 how to compensate this.

\section{B. Lie transformations pseudogroup}

Consider the pseudogroup of Lie transformations of $M=J_{r}^{k}(\mathfrak{M})$. It consists of local diffeomorphisms of the jet-bundle, preserving the Cartan distribution $\mathcal{C}_{k}$. This pseudogroup $G$ is integrable. We will calculate its symbols below.

To simplify we consider the corresponding Lie pseudogroup of vector fields. A vector field is called an infinitesimal Lie transformation if its flow is a local Lie transformation. Since we will work locally, there will be no distinction between $J_{r}^{k}(\mathfrak{M})$ and its local chart. Thus we will write $M=J^{k} \pi$ for simplicity, where $\pi: E_{\pi} \rightarrow B$ is a vector bundle.

\section{B.1. Lifts of point transformations}

In the case $r>1$, denote the projection of the Lie field to $J^{\epsilon} \pi=J^{0} \pi$ by $X=\sum_{i} a^{i}(x, u) \partial_{x^{i}}+\sum_{j} b^{j}(x, u) \partial_{u^{j}}$. Then the prolongation to $J^{k} \pi$ is

$$
X^{(k)}=\sum_{i} a^{i}(x, u) \mathcal{D}_{i}^{(k+1)}+\sum_{j ;|\sigma| \leq k} \mathcal{D}_{\sigma}\left(\varphi^{j}\right) \partial_{p_{\sigma}^{j}},
$$

where $\varphi^{j}=b^{j}-\sum_{i=1}^{n} a^{i} p_{i}^{j}$ are components of the so-called generating function $\varphi=\left(\varphi^{1}, \ldots, \varphi^{r}\right)$ and $\mathcal{D}_{i}^{(k+1)}=\partial_{x^{i}}+\sum_{j ;|\sigma| \leq k} p_{\sigma+1_{i}}^{j} \partial_{p_{\sigma}^{j}}$ is the operator of total derivative restricted to $J^{k} \pi$. Though the coefficients of (15) depend seemingly on the $(k+1)$-jets, the Lie field is in fact on $J^{k} \pi$.

Formula (15) follows from the claim the Lie field preserves the co-distribution

$$
\operatorname{Ann}\left(\mathcal{C}_{k}\right)=\left\langle\omega_{\sigma}^{j}=d p_{\sigma}^{j}-\sum_{i} p_{\sigma+1_{i}}^{j} d x^{i}|1 \leq j \leq r,| \sigma \mid<k\right\rangle
$$

and the formula $d=\sum_{i} d x^{i} \otimes \mathcal{D}_{i}^{(k+1)}+\sum_{j ;|\sigma| \leq k} \omega_{\sigma}^{j} \otimes \partial_{p_{\sigma}^{j}}$ on $J^{k} \pi$.

Proposition 24. The l-symbol $\mathfrak{g}^{l}\left(x_{k}\right)$ of the pseudogroup $G$ at a point $x_{k} \in J^{k} \pi$ admits the splitting $\mathfrak{g}^{l}=\mathfrak{g}_{H}^{l} \oplus \mathfrak{g}_{V}^{l}$ depending on a point $x_{k+1} \in F\left(x_{k}\right)$. The horizontal part is isomorphic to

$$
\mathfrak{g}_{H}^{l}\left(x_{k}\right) \simeq\left[S^{l} \nu_{x_{0}}^{*} \oplus \sum_{0<i<k}\left(S^{i} \tau_{x}^{*} \otimes S^{l-1} \nu_{x_{0}}^{*}\right) \oplus \sum_{i \geq k}\left(S^{i} \tau_{x}^{*} \otimes S^{k+l-i-1} \nu_{x_{0}}^{*}\right)\right] \otimes \tau_{x},
$$

while the vertical (evolutionary) parts is represented as

$$
\mathfrak{g}_{V}^{l}\left(x_{k}\right) \simeq\left[\sum_{0 \leq i<k}\left(S^{i} \tau_{x}^{*} \otimes S^{l} \nu_{x_{0}}^{*}\right) \oplus \sum_{i \geq k}\left(S^{i} \tau_{x}^{*} \otimes S^{k+l-i} \nu_{x_{0}}^{*}\right)\right] \otimes \nu_{x_{0}} .
$$


Proof. The space $T_{x_{k}} J^{k} \pi$ is decomposed into direct sum of the horizontal $L\left(x_{k+1}\right) \subset \mathcal{C}_{k}\left(x_{k}\right)$ and the vertical $T_{x_{k}}^{v}=\operatorname{Ker}\left(\pi_{k}\right)_{*}$ components. Thus we have:

$\mathfrak{g}^{l}\left(x_{k}\right) \subset S^{l} T_{x_{k}}^{*} J^{k} \pi \otimes T_{x_{k}} J^{k} \pi=\left[S^{l} T_{x_{k}}^{*} J^{k} \pi \otimes L\left(x_{k+1}\right)\right] \oplus\left[S^{l} T_{x_{k}}^{*} J^{k} \pi \otimes T_{x_{k}}^{v} J^{k} \pi\right]$,

whence the required splitting. In formula (15) the horizontal and vertical components correspond to the first and the second summands respectively.

Denote by $\mu_{a}$ the ideal in $C^{\infty}(B)$ generated by functions vanishing at $a \in B$, and by $\mu_{a}^{l}$ its degree. Let $\mu_{a}^{l}(\mathfrak{L i \mathfrak { e }})$ be the space of Lie fields vanishing at $a$ to the order $l$. Then $\mathfrak{g}^{l}\left(x_{k}\right)=\mu_{x_{k}}^{l}(\mathfrak{L i e}) / \mu_{x_{k}}^{l+1}(\mathfrak{L i e})$.

As in the contact and symplectic cases we represent the symbol via the jets of generating functions. It embeds into the space $S^{l} T_{x_{k}}^{*} J^{k} \pi \otimes T_{x_{k}} J^{k} \pi$ by (15).

Let us choose a coordinate system such that the point $x_{k}$ becomes the origin. If $x_{k}=[s]_{a}^{k}$ for some section $s$, this is achieved by making it the zero-section: $s=\left\{u^{j}=0\right\}$. Then the condition $X^{(k)} \in \mu_{x_{k}}^{l}$ is expressed via the components of the generating function as follows:

$$
a^{i} \in \mu_{x_{0}}^{l}, \partial_{x^{\sigma}}\left(a^{i}\right) \in \mu_{x_{0}}^{l-1}, \quad b^{j} \in \mu_{x_{0}}^{l}, \partial_{x^{\sigma}}\left(b^{j}\right) \in \mu_{x_{0}}^{l}, \quad 0 \leq|\sigma| \leq k .
$$

This yields the claim. Note that the decomposition $T_{x_{0}}^{*} J^{0} \pi=\tau_{x}^{*} \oplus \nu_{x_{0}}^{*}$ is induced by the point $x_{1}$ and so the representation in the statement is canonical.

\section{B.2. Lifts of contact transformations}

A Lie transformation for $r=1$ is determined by a contact transformation $X^{(1)}=X_{\varphi}$ on $J^{1} \pi$ with a generating scalar-valued function $\varphi=\varphi\left(x^{i}, u, p_{i}\right)$ :

$$
X^{(1)}=\sum_{i}\left[\mathcal{D}_{i}^{(1)}(\varphi) \partial_{p_{i}}-\partial_{p_{i}}(\varphi) \mathcal{D}_{i}^{(1)}\right]+\varphi \partial_{u}
$$

The prolongation of this field to $J^{k} \pi$ is given by the formula similar to (15):

$$
X^{(k)}=-\sum_{i} \partial_{p_{i}}(\varphi) \mathcal{D}_{i}^{(k+1)}+\sum_{|\sigma| \leq k} \mathcal{D}_{\sigma}^{(k)}(\varphi) \partial_{p_{\sigma}} .
$$

Again a calculation shows this is a field on $J^{k} \pi$, coinciding with $X_{\varphi}$ for $k=1$.

We will need below a decomposition $T_{x_{1}} J^{1} \pi=\tau_{x} \oplus \nu_{x_{0}} \oplus v_{x_{1}}$, which is not canonical. Though the point $x_{2}$ determines the splitting $T_{x_{1}} J^{1} \pi=L\left(x_{1}\right) \oplus T_{x_{1}}^{v}$, the last summand is further decomposed by a connection in the bundle $\pi_{1,0}$.

Proposition 25. The l-symbol of the pseudogroup $G$ at a point $x_{k} \in J^{k} \pi$ is

$$
\begin{array}{r}
\mathfrak{g}^{l}\left(x_{k}\right) \simeq \sum_{0 \leq j \leq l}\left(S^{j} \nu_{x_{0}}^{*} \otimes S^{l+1-j} v_{x_{1}}^{*}\right) \oplus S^{l} \nu_{x_{0}}^{*} \oplus \sum_{1 \leq i<k ; j}\left(S^{i} \tau_{x}^{*} \otimes S^{j} \nu_{x_{0}}^{*} \otimes S^{l-j} v_{x_{1}}^{*}\right) \\
\oplus \sum_{k \leq i ; j}\left(S^{i} \tau_{x}^{*} \otimes S^{j} \nu_{x_{0}}^{*} \otimes S^{k+l-i-j} v_{x_{1}}^{*}\right) .
\end{array}
$$


Proof. As in proposition 24] due to (16), in the coordinate system $\left(x^{i}, u\right)$ such that $p_{\sigma}\left(x_{k}\right)=0$ for $|\sigma| \leq k$, the condition $X^{(k)} \in \mu_{x_{k}}^{l}(\mathfrak{L i e})$ is equivalent to:

$$
\varphi \in \mu_{x_{1}}^{l}, \partial_{p_{i}}(\varphi) \in \mu_{x_{1}}^{l}, \partial_{x^{\sigma}}(\varphi) \in \mu_{x_{1}}^{l}, \quad 0 \leq|\sigma| \leq k .
$$

The claim follows.

\section{References}

[AB] M. Auslander, D. A. Buchsbaum, Codimension and multiplicity, Annals of Math. 68, no. 3 (1958), 625-657.

[AG] V. Arnold, A. Givental, Symplectic geometry, (Russian) Current problems in mathematics. Fundamental directions, 4, no. 291, Itogi Nauki i Tekhniki, AN SSSR, VINiTI, Moscow, (1985), 5-139. Engl. transl.: Dynamical systems - IV, Encyclopaedia Math. Sci., 4, Springer, Berlin, (2001), 1-138.

[BH] W. Bruns, J. Herzog, Cohen-Macaulay rings, Cambridge University Press, Cambridge, U.K. (1993)

[BM] C. Buttin, P. Molino, Theoreme general d'equivalence pour les pseudogroupes de Lie plats transitifs, J. Diff. Geometry, 9 (1974), 347-354.

[C1] E. Cartan, Sur la structure des groupes infinis de transformations, in Oeuvres completes, II 2, 571-714, Gautier-Villars, Paris (1953).

[C2] E. Cartan, Sur les varietes a connexion projective, Bull. Soc. Math. France 52 (1924), 205-241.

[CM] S. S. Chern, J. Moser Real hypersurfaces in complex manifolds, Acta Math., 133 (1974), 219-271.

[E] C. Ehresmann, Introduction à la théorie des structures infinitésimales et des pseudogroupes de Lie, Colloque de topologie et geometrie differentielle, Strasbourg (1952), no. 11, $16 \mathrm{pp}$.

[Go] H. Goldschmidt, Integrability criteria for systems of nonlinear partial differential equations, J. Diff. Geom., 1(3) (1967), 269-307.

[GS1] V. Guillemin, S. Sternberg, An algebraic model of transitive differential geometry, Bull. A.M.S., 70 (1964), 16-47.

[GS2] V. Guillemin, S. Sternberg Deformation theory of pseudogroup structures, Mem. A.M.S., 64 (1966), 1-80.

[Gu] V. Guillemin, The integrability problem for G-structures, Trans. A.M.S., 116 (1965), 544-560.

[H] M. Halpen, Sur les invariants differentiels, Thèse, Paris (1878).

[Ko] S. Kobayashi, Transformation groups in Differential geometry, Springer-Verlag (1972). 
[Kr1] B.S. Kruglikov, Nijenhuis tensors and obstructions for pseudoholomorphic mapping constructions, Math. Notes, 63, issue 4 (1998), 541-561.

[Kr2] B.S. Kruglikov, Non-existence of higher-dimensional pseudoholomorphic submanifolds, Manuscripta Mathematica (2003) 111 (2003), 51-69.

[Kr3] B.S. Kruglikov, Classification of Monge-Ampere equations with two variables, in: Geometry and topology of caustics - CAUSTICS '98 (Warsaw); Banach Center Publications, 50 (1999), 179-194.

[KL1] B.S. Kruglikov, V. V. Lychagin, On equivalence of differential equations, Acta et Comment. Univ. Tartuensis Math. 3 (1999), 7-29.

[KL2] B.S. Kruglikov, V.V. Lychagin, Transitive and transversal actions of pseudogroups on submanifolds, Russian Math. (Izv. VUZ, Matematika) 48, no. 11, 30-44 [engl. 27-40] (2004).

[KL3] B. S. Kruglikov, V.V. Lychagin, Spencer $\delta$-cohomologies, restrictions and involutive symbolic PDEs, ArXiv: math.DG/0503124

[KLV] I.S. Krasilschik, V.V. Lychagin, A. M. Vinogradov, Geometry of jet spaces and differential equations, Gordon and Breach (1986).

[Kum] A. Kumpera, Invariants differentiels d'un pseudogroupe de Lie. I-II. J. Differential Geometry 10 (1975), no. 2, 289-345; 10 (1975), no. 3, 347-416.

[KS] A. Kumpera, D. Spencer, Lie equations. Volume 1: General theory, Princeton University Press and University Tokyo Press (1972).

[Kur] M. Kuranishi, On the local theory of continuous infinite pseudo groups. I-II. Nagoya Math. J., 15 (1959), 225-260; 19 (1961), 55-91.

[KLR] A. Kushner, V. Lychagin, V. Roubtsov, Contact geometry and non-linear differential equations, Cambridge University Press (2006).

[La] E. P. Lane, A treatise on projective differential geometry, University of Chicago Press (1942).

[Lib] P. Libermann, Pseudogroupes infinitésimaux de Lie, C. R. Acad. Sci. Paris, 246 (1958), 531-534.

[Li1] S. Lie, Theorie der Transformationsgruppen (Zweiter Abschnitt, unter Mitwirkung von Prof.Dr.Friederich Engel), Teubner, Leipzig (1890).

[Li2] S. Lie, Ueber Differentialinvarianten, Math. Ann. 24 (1884), no. 4, 537-578.

[Li3] S. Lie, Verwertung des Gruppenbegriftes für Differentialgleichungen, I, Leipzig Ber. 47 (1895), 261-322; Gesam. Abh. Bd. VI, 539-591.

[Li4] S. Lie, Zur Invariantenteorie der Gruppe der Bewgungen, Leipzig Ber. 48 (1896), 466-477; Gesam. Abh. Bd. VI, 639-648.

[Li5] Sophus Lie's 1884 differential invariant paper. Translated from the German by M. Ackerman. Comments by Robert Hermann. Lie Groups: History, Frontiers and Applications, Vol. III. Math Sci Press, Brookline, Mass. (1976). 
[Ly] V. Lychagin, Homogeneous geometric structures and homogeneous differential equations, A.M.S. Transl. Ser., The interplay between differential geometry and differential equations, ser. 2, 167 (1995), 143-164.

[M] B. Malgrange, Equations de Lie. I-II. J. Differential Geometry, 6 (1972), 503522; 7 (1972), 117-141.

[Mum] D. Mumford, Algebraic geometry I. Complex projective varieties, SpringerVerlag (1976).

[MMR] J. Munoz, F. J. Muriel, J. Rodriguez, On the finiteness of differential invariants, J. Math. Anal. Appl. 284 (2003), no. 1, 266-282.

[Ol] P. Olver, Applications of Lie groups to differential equations, Graduate Texts in Mathematics, 107, Springer-Verlag, New York (1986).

[Ov] L. V. Ovsiannikov, Group analysis of differential equations, Russian: Nauka, Moscow (1978); Engl. transl.: Academic Press, New York (1982).

[P] A.S. Pollack, The integrability problem for pseudogroup structures, J. Diff. Geometry, 9 (1974), 355-390.

[SS] I.M. Singer, S. Sternberg, On the infinite groups of Lie and Cartan, J. d'Analyse Math., 15 (1965), 1-114.

[S] D. Spencer, Deformation of structures on manifolds defined by transitive pseudogroups, Ann. of Math., 76, no.2 (1962), 306-445.

[Ta] N. Tanaka, On differential systems, graded Lie algebras and pseudogroups, Jour. Math. Kyoto Univ., 10 (1970), 1-82.

[Tr1] A. Tresse, Sur les invariants differentiels des groupes continus de transformations, Acta Math. 18 (1894), 1-88.

[Tr2] A. Tresse, Détermination des invariants ponctuels de l'équation différentielle ordinaire de second ordre $y^{\prime \prime}=\omega\left(x, y, y^{\prime}\right)$, Mémoire couronné par l'Académie Jablonowski; S. Hirkel, Leipzig (1896).

[V] E. Vessiot, Sur l'integration des systèms différentiels qui admettent des groupes continus de transformations, Acta Math. 28 (1904), 307-350.

[W] E. J. Wilczynski, "Projective differential geometry of curves and ruled surfaces, Leipzig: Teubner (1906). 